\title{
Dynamics of fluid vesicles in shear flow: Effect of membrane viscosity and thermal fluctuations
}

\author{
Hiroshi Noguchi* and Gerhard Gompper ${ }^{\dagger}$ \\ Institut für Festkörperforschung, Forschungszentrum Jülich, 52425 Jülich, Germany \\ (Received 6 December 2004; revised manuscript received 22 February 2005; published 1 July 2005)
}

\begin{abstract}
The dynamical behavior of vesicles is investigated in simple shear flow. A simulation technique is presented that combines a three-dimensional particle-based mesoscopic model (multiparticle collision dynamics) for the solvent with a dynamically triangulated surface model for the membrane. In this model, thermal fluctuations of the solvent and of the membrane are consistently taken into account. The membrane viscosity can be varied by changing the bond-flip rate of the dynamically triangulated surface. Vesicles are found to transit from steady tank-treading to unsteady tumbling motion with increasing membrane viscosity. At small reduced volumes, the shear induces a transformation from a discocyte to a prolate shape at low membrane viscosity. On the other hand, at high membrane viscosity, the shear induces a transformation from prolate to discocyte, or tumbling motion accompanied by shape oscillations between these two states. Thermal fluctuations induce intermittent tumbling and smooth out the transitions. This effect can be understood from a simplified stochastic model.
\end{abstract}

DOI: 10.1103/PhysRevE.72.011901

\section{INTRODUCTION}

Vesicles are closed bilayer membranes, which show a rich variety of different morphologies depending on the lipid architecture as well as their environment. In thermal equilibrium, vesicle shapes have been investigated intensively on the basis of a curvature-elastic model and are now understood very well $[1,2]$. In comparison, the behavior in nonequilibrium situations is much less explored. The behavior of vesicles in flow fields is a paradigmatic example for the richness of dynamical phenomena out of thermal equilibrium. For example, the formation of very long tubular vesicles [3] and the lifting of a vesicle from a wall [4-6] have been observed in shear flows.

Synthetic lipid-bilayer vesicles are very nice model systems to study the effect of flow fields on the behavior of soft mesoscopic particles. The results obtained for these systems will help to understand more complex systems, like the behavior of cells in blood vessels. For example, red blood cells have been found to form parachute shapes in microvessel or capillary flow [7-10]. Some diseases, such as diabetes mellitus and sickle cell anemia, reduce the deformability of red blood cells, and the cells often block microvascular flow $[11,12]$. Thus, the deformability of cells and vesicles is an important subject not only of fundamental research but also in medical applications.

The dynamical behavior of vesicles in simple shear flow has been studied experimentally [11-15], theoretically [16-19], and numerically [20-22]. The vesicle shape is determined by the competition of the curvature elasticity of the membrane, the constraints of constant volume $V$ and constant surface area $S$, and the external hydrodynamic forces. One of the difficulties in theoretical studies of the hydrodynamic effects on vesicle dynamics is the boundary condition for the embedding fluid on the vesicle surface, which changes its shape dynamically. In some previous studies, a fluid vesicle

\footnotetext{
*Email address: hi.noguchi@fz-juelich.de

†Email address: g.gompper@fz-juelich.de
}

PACS number(s): 87.16.Dg, 83.80.Lz, 87.17.Aa

was therefore modeled as an ellipsoid with fixed shape [16]. More recently, the time evolution of the shape was studied numerically using a boundary integral method in three spatial dimensions $[5,20]$ or an advected-field method in two dimensions [21,22]. Red blood cells have a spectrin network attached to their plasma membrane, which helps to retain the integrity of the cell in strong shear gradients or capillary flow. Due to the spectrin network, the red blood cell membrane has a nonzero shear modulus, and has therefore been modeled as an elastic capsule of discocyte shape. The deformation of spherical capsules has been studied, both analytically and numerically, with various elastic laws without bending resistance $[23,24]$. discocyte capsules with bending resistance have been investigated numerically with the boundary integral method $[25,26]$. It is important to point out that there are fundamental differences between the flow behaviors of fluid vesicles and red blood cells, which are due to the shear elasticity of the spectrin network.

Two types of dynamics have been found in these studies, a steady state with a tank-treading motion of the membrane and a finite inclination angle with the flow direction, and an unsteady state with a tumbling (flipping) motion. For fluid vesicles, the transition from tank-treading to tumbling motion with increasing viscosity of the internal fluid has been studied in Refs. [16,22].

The membrane viscosity is an important factor for vesicle dynamics in shear flow. For example, the membrane of red blood cells becomes more viscous on aging $[18,27]$ or in diabetes mellitus [9]. Experiments indicate that the energy dissipation in the membrane is larger than that inside a red blood cell $[18,19]$. Furthermore, it has been shown recently that vesicles can be made not only from lipid bilayers, but also from bilayers of block copolymers [28]. The membrane viscosity of these "polymersomes" is several orders of magnitude larger than for liposomes, and can be changed over a wide range by varying the polymer chain length [29].

Theoretically, the model of Keller and Skalak [16] for vesicles of fixed ellipsoidal shape has been generalized to describe the effects of membrane viscosity [18]. The behavior of liquid drops with surface viscosity and variable shape has been studied numerically by the boundary integral 
method [30]. However, the effect of the membrane viscosity on the behavior of deformable vesicles with fixed surface area has not been investigated so far.

Several particle-based simulation techniques for fluid flow have been developed in recent years, such as direct simulation Monte Carlo [31,32], smooth particle hydrodynamics $[33,34]$, and dissipative particle dynamics $[35,36]$. In order to investigate vesicle hydrodynamics, we recently proposed a combination of a mesoscopic model for the solvent and a coarse-grained, dynamically triangulated surface model for the membrane [37]. This approach has four main advantages: (i) The membrane is described explicitly, so that its properties like viscosity or composition can be varied easily; (ii) thermal fluctuations of both the solvent and the membrane are fully and consistently taken into account; (iii) the method can easily be generalized to more complex flow geometries; and (iv) no numerical instabilities can occur. We employ a particle-based hydrodynamics method [38-53] to simulate the solvent, which is called multiparticle collision dynamics (MPCD) [42,50,52] or stochastic rotation dynamics $[41,45,48]$. This method was applied, for example, to flow around a solid obstacle $[42,43,53]$, to polymer dynamics in thermal equilibrium [40,50], and to ternary amphiphilic fluids [44].

With this model, we study the dynamics of fluid vesicles with viscous membranes in simple shear flow. We describe the model and the simulation procedure in detail in Sec. II. Our results for the vesicle shape, the tank-treading frequency, the inclination angle between the vesicle axis and the flow direction, and the transitions between discocyte, prolate, and stomatocyte vesicle shapes are presented in Sec. III. In Sec. IV, we derive a simplified stochastic model, which can be used to understand the simulation results. In particular, we discuss the effect of thermal fluctuations on vesicle shape, shape transitions, tank treading, and tumbling.

\section{METHOD AND MODEL}

\section{A. Multiparticle collision dynamics of the solvent}

In multiparticle collision dynamics, the solvent is described by $N_{\mathrm{s}}$ pointlike particles of mass $m_{\mathrm{s}}$ moving in a rectangular box of size $L_{x} \times L_{y} \times L_{z}$. The algorithm consists of alternating streaming and collision steps. In the streaming step, the particles move ballistically and the position of each particle $\mathbf{r}_{i}$ is updated according to

$$
\mathbf{r}_{i}\left(t+\Delta t_{\mathrm{CD}}\right)=\mathbf{r}_{i}(t)+\mathbf{v}_{i}(t) \Delta t_{\mathrm{CD}}
$$

where $\mathbf{v}_{i}$ is the velocity of particle $i$ and $\Delta t_{\mathrm{CD}}$ is the time interval between collisions. In the collision step, the particles are sorted into cubic cells of lattice constant $a$. The collision step consists of a stochastic rotation of the relative velocity of each particle in a cell,

$$
\mathbf{v}_{i}^{(\text {new })}(t)=\mathbf{v}_{\mathrm{cm}}(t)+\boldsymbol{\Omega}(\varphi)\left[\mathbf{v}_{i}(t)-\mathbf{v}_{\mathrm{cm}}(t)\right],
$$

where $\mathbf{v}_{\mathrm{cm}}$ is the velocity of the center of mass of all particles in the cell. The matrix $\boldsymbol{\Omega}(\varphi)$ rotates velocities by a fixed angle $\varphi$ around an axis, which is chosen randomly for each cell [39]. In our simulation, the angle $\varphi=\pi / 2$ is employed.
The momentum and energy are conserved in this procedure. We apply a random-shift procedure [41] before each collision step to ensure Galilean invariance.

In experimental conditions of red blood cells and liposomes in shear flow with usual shear rates $\dot{\gamma}$, the Reynolds number $\operatorname{Re}=\dot{\gamma} \rho R_{0}^{2} / \eta_{0}$ is very small, typically $\operatorname{Re} \sim 10^{-3}$. Here, $R_{0}=\sqrt{S / 4 \pi}$ is the effective vesicle radius, $\rho$ is the mass density of the solvent, and $\eta_{0}$ is the solvent viscosity. It has been shown in Ref. [50] that the description of hydrodynamics at low Reynolds numbers and large Schmidt numbers in MPCD requires small mean free paths $\lambda=\Delta t_{\mathrm{CD}} \sqrt{k_{\mathrm{B}} T / m_{\mathrm{s}}}$, where $k_{\mathrm{B}}$ is the Boltzmann constant and $T$ is the temperature. Therefore, we choose $\lambda / a=0.025$. For the employed particle density of $\rho=10 m_{\mathrm{s}} / a^{3}$ the viscosity of solvent fluid is then found to be $[47,48]$

$$
\eta_{0}=\left[\frac{n-1}{18} \frac{a}{\lambda}+\frac{n(2 n+3)}{6(n-1)} \frac{\lambda}{a}\right] \sqrt{m_{\mathrm{s}} k_{\mathrm{B}} T} / a^{2}=20.1 \sqrt{m_{\mathrm{s}} k_{\mathrm{B}} T} / a^{2}
$$

where $n$ is the number of particles in a cell. This implies a reasonably small Reynolds number of $\operatorname{Re} \simeq 0.1$ for the simulated vesicle size of $R_{0} / a=5.68$.

To induce a shear flow, we employed Lees-Edwards boundary condition $[48,54]$, which gives a linear flow profile $\left(v_{x}, v_{y}, v_{z}\right)=\left(\dot{\gamma}_{z}, 0,0\right)$ in the MPCD fluid. We simulated the vesicle dynamics in a simulation box with $L_{x}=50 a$ and $L_{y}$ $=L_{z}=30 a$. With $\rho=10 m_{\mathrm{s}} / a^{3}$, this implies a total number $N_{\mathrm{s}}$ $=450000$ of solvent particles.

\section{B. Triangulated-surface model of the membrane}

For the membrane, we employ a dynamically triangulated surface model $[55,56]$, in which the membrane is described by $N_{\mathrm{mb}}$ vertices which are connected by tethers to form a triangular network of spherical topology. The vertices have excluded volume and mass $m_{\mathrm{mb}}$. In the current simulations, we consider $N_{\mathrm{mb}}=500$ and $m_{\mathrm{mb}}=10 m_{\mathrm{s}}$. The shapes and fluctuations of the membrane are controlled by curvature elasticity, with the Hamiltonian [57]

$$
U_{\mathrm{cv}}=\frac{\kappa}{2} \int\left(C_{1}+C_{2}\right)^{2} d S,
$$

where $\kappa$ is the bending rigidity and $C_{1}$ and $C_{2}$ are the principal curvatures at each point of the membrane.

The curvature energy is discretized as $[58,59]$

$$
U_{\mathrm{cv}}=\frac{\kappa}{2} \sum_{i} \frac{1}{\sigma_{i}}\left\{\sum_{j(i)} \frac{\sigma_{i, j} \mathbf{r}_{i, j}}{r_{i, j}}\right\}^{2},
$$

where the sum over $j(i)$ is over the neighbors of a vertex $i$ that are connected by tethers. The bond vector between the vertices $i$ and $j$ is $\mathbf{r}_{i, j}$, and $r_{i, j}=\left|\mathbf{r}_{i, j}\right|$. The length of a bond in the dual lattice is $\sigma_{i, j}=r_{i, j}\left[\cot \left(\theta_{1}\right)+\cot \left(\theta_{2}\right)\right] / 2$, where the angles $\theta_{1}$ and $\theta_{2}$ are opposite to bond $i j$ in the two triangles sharing this bond. Finally, $\sigma_{i}=0.25 \Sigma_{j(i)} \sigma_{i, j} r_{i, j}$ is the area of the dual cell of vertex $i$. We use $\kappa=20 k_{\mathrm{B}} T$, which is a typical value for lipid bilayers.

In contrast to previous Monte Carlo simulations of dynamically triangulated surfaces, we employ a Stillinger- 
Webber potential [60] for bond $\left(U_{\text {bond }}\right)$ and excluded-volume $\left(U_{\text {rep }}\right)$ interactions between vertices. These smooth potentials make the model amenable for molecular dynamics simulations. Vertices connected by a tether interact via the attractive bond potential

$$
U_{\text {bond }}\left(r_{i, j}\right)= \begin{cases}\frac{b \exp \left[1 /\left(l_{\mathrm{c} 0}-r_{i, j}\right]\right.}{l_{\max }-r_{i, j}} & \left(r_{i, j}>l_{\mathrm{c} 0}\right), \\ 0 & \left(r_{i, j} \leqslant l_{\mathrm{c} 0}\right),\end{cases}
$$

while all vertices repel each other at short distances via the excluded-volume potential

$$
U_{\text {rep }}\left(r_{i, j}\right)= \begin{cases}\frac{b \exp \left[1 /\left(r_{i, j}-l_{\mathrm{c} 1}\right)\right]}{r_{i, j}-l_{\min }} & \left(r_{i, j}<l_{\mathrm{c} 1}\right), \\ 0 & \left(r_{i, j} \geqslant l_{\mathrm{c} 1}\right) .\end{cases}
$$

In the Hamiltonian, the potentials $U_{\text {bond }}$ and $U_{\text {rep }}$ are summed over all bonds and over all vertex pairs, respectively. In Eqs. (6) and (7), $l_{\max }=1.33 a$ is the maximum bond length, and $l_{\min }=0.67 a$ is the minimum distance between any two vertices. We use $b=80 k_{\mathrm{B}} T$, and the cutoff lengths $l_{\mathrm{c} 0}=1.15 a$ and $l_{\mathrm{c} 1}=0.85 a$. These potentials are smoothly connected at the cutoff lengths, with $d^{n} U_{\text {bond }}(r) / d r^{n}=0$ at $r=l_{c} 0$ for all $n$, and similarly for $U_{\text {rep }}(r)$ at $r=l_{c 1}$. Newton's equations of motion for the vertices are integrated by the velocity-Verlet algorithm with time steps $\Delta t_{\mathrm{MD}}$.

To model the fluidity of the membrane, tethers can be flipped between the two possible diagonals of two adjacent triangles. A number $\psi N_{\mathrm{b}}$ of bond-flip attempts is performed with the METROPOLIS Monte Carlo method [59] at time intervals $\Delta t_{\mathrm{BF}}$, where $N_{\mathrm{b}}=3\left(N_{\mathrm{mb}}-2\right)$ is the number of bonds in the network, and $0<\psi<1$ is a parameter of the model. The flat region around $r_{i, j}=a$ of the total potential between neighboring vertices allows a sufficiently large acceptance rate $\Gamma$ $=10^{-2}$ of the bond-flip procedure.

In the absence of external forces and without coupling to the solvent, the average area of a triangle is well approximated by the area $\sqrt{3} a^{2} / 4$ of an equilateral triangle with edge length $a$. Fluctuations of the area are small [59]. In general, however, the enclosed volume $V$ and the surface area $S$ of a vesicle are affected by the competition of the curvature energy of the membrane, thermal fluctuations, the pressure difference between the internal and external fluids, and forces induced by shear flows. Therefore, in order to keep the surface area $S$ and the volume $V$ constant and the densities of interior and exterior solvents the same to high accuracy, in particular in the presence of external forces, we add constraint potentials

$$
\begin{gathered}
U_{\mathrm{S}}=\frac{1}{2} k_{\mathrm{S}}\left(S-S_{0}\right)^{2}, \\
U_{\mathrm{V}}=\frac{1}{2} k_{\mathrm{V}}\left(V-V_{0}\right)^{2}
\end{gathered}
$$

to the Hamiltonian. Here we use the parameters $k_{\mathrm{S}}=1, k_{\mathrm{V}}$ $=0.5$, and $S_{0}=0.41 a^{2} N_{\text {tri }}$ with the number of triangles $N_{\text {tri }}$ $=2\left(N_{\mathrm{mb}}-2\right)$ on the vesicle. With these potentials, the volume $V$ and surface area $S=405 a^{2}$ of a vesicle are kept constant

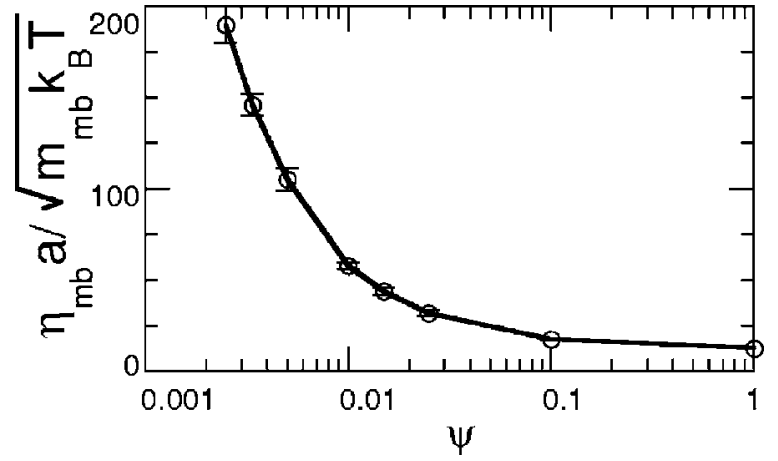

FIG. 1. Dependence of the membrane viscosity $\eta_{\mathrm{mb}}$ on the probability $\psi$ for the selection of a bond for a bond-flip attempt.

with a deviation of less than $1 \%$ of the target values for all simulated systems.

The bond flips provide a very convenient way to vary the membrane viscosity $\eta_{\mathrm{mb}}$, which increases with decreasing probability $\psi$ for the selection of a bond for a bond-flip attempt. We have determined the membrane viscosity quantitatively from a simulation of a flat membrane in twodimensional Poiseuille flow. The triangulated membrane is put in a rectangular box of size $L_{x} \times L_{y}$ with periodic boundary conditions in the $x$ direction. The edge vertices at the lower and upper boundary $\left(y= \pm L_{y} / 2\right)$ are fixed at their positions. A gravitational force $\left(m_{\mathrm{mb}} g, 0\right)$ is applied to all membrane vertices to induce a flow. Rescaling of relative velocities is employed as a thermostat. We study a membrane with $N_{\mathrm{mb}}=1860$ vertices, total area $S=0.41 a^{2} N_{\text {tri }}$, and $L_{x} / a$ $=55.2$. Then, the membrane viscosity is calculated from $\eta_{\mathrm{mb}}=\rho_{\mathrm{mb}} g L_{y} / 8 v_{\max }$, where $\rho_{\mathrm{mb}}$ is average mass density of the membrane particles, and $v_{\max }$ the maximum velocity of the parabolic flow profile. The membrane viscosity $\eta_{\mathrm{mb}}$, which is obtained in this way, is shown in Fig. 1. As $\psi$ decreases, it takes longer and longer for a membrane particle to escape from the cage of its neighbors, and $\eta_{\mathrm{mb}}$ increases. This is very similar to the behavior of a hard-sphere fluid with increasing density. Finally, for $\psi=0$, the membrane becomes solid.

The vesicle is kept at the center of the simulation box by shifting the positions of the vesicle and solvent particles with finite time steps $\Delta t_{\mathrm{BS}}$ to keep the vesicle far from the LeesEdwards boundaries. The relative velocities in the collision cells are rescaled after time steps $\Delta t_{\mathrm{CD}}$ in order to keep the temperature constant. The fluids in the interior and exterior of the vesicle are taken to be the same, in particular to have the same viscosity $\eta_{0}$.

\section{Interaction between membrane and mesoscopic solvent}

For membrane viscosity $\eta_{\mathrm{mb}}>0$, we let solvent particles interact with the membrane in two ways to obtain nonslip boundary conditions. First, the membrane vertices are included in the MPCD collision procedure, as suggested for polymers in Ref. [40]. Second, the solvent particles are scattered with a bounce-back rule from the membrane surface.

We have investigated two types of membrane-solvent interactions to prevent solvent particles from penetrating the 
membrane. The first method is to place hard spheres on the membrane vertices. The radius of the spheres is chosen to be large enough for the spheres to overlap and to completely cover the whole membrane surface. It turns out quickly that this method does not work for the vesicle sizes of a few hundred vertices we are currently able to simulate. The reason is that the surface area accessible to the interior solvent particles is much smaller than the surface area exposed to the exterior solvent. A simple estimate shows that this area difference $\Delta S$ is approximately $2 S_{0} \delta / R_{0}$, where $\delta$ is the effective membrane thickness and $R_{0}$ the vesicle radius. This area difference implies that at the same solvent density inside and outside the vesicle, which corresponds to an ideal-gas pressure $p$, there is a strong compressive force $p \Delta S$. This force acts to increase the interior solvent density until the number of solvent collisions on both sides of the membrane are the same. This density difference $\delta \rho$ implies a total expansive force of $\Delta \rho k_{\mathrm{B}} T S_{0}$. By equating these two forces, we find $\Delta \rho / \rho=2 \delta / R_{0}$.

In order to avoid this difficulty, we introduce a second model in which the membrane triangles have a finite but very small thickness $\delta=2 l_{\mathrm{BS}}$. For the bounce-back collisions of the solvent with the membrane, we use a similar procedure as suggested in Ref. [49] for a spherical particle embedded in a MPCD solvent. The scattering process is performed at discrete time steps $\Delta t_{\mathrm{BS}}$. Therefore, scattering does not occur exactly on the membrane surface, but the solvent particles can penetrate into the membrane film. We distinguish the solvent particles inside $\left(1 \leqslant i \leqslant N_{\text {in }}\right)$ and outside $\left(N_{\text {in }}<i\right.$ $\leqslant N_{\mathrm{s}}$ ) of the vesicle. Particles that enter the membrane film, i.e., which have a distance to the triangulated surface smaller than $l_{\mathrm{BS}}$, or interior particles which reach the exterior volume, and vice versa, are scattered at the membrane triangle with the closest center of mass. Explicitly,

$$
\begin{aligned}
& \mathbf{v}_{\mathrm{s}}^{(\text {new) }}(t)=\mathbf{v}_{\mathrm{s}}(t)-\frac{6 m_{\mathrm{mb}}}{m_{\mathrm{s}}+3 m_{\mathrm{mb}}}\left[\mathbf{v}_{\mathrm{s}}(t)-\mathbf{v}_{\mathrm{tri}}(t)\right], \\
& \mathbf{v}_{\text {tri }}^{\text {(new) }}(t)=\mathbf{v}_{\text {tri }}(t)+\frac{2 m_{\mathrm{s}}}{m_{\mathrm{s}}+3 m_{\mathrm{mb}}}\left[\mathbf{v}_{\mathrm{s}}(t)-\mathbf{v}_{\text {tri }}(t)\right],
\end{aligned}
$$

when $\left[\mathbf{v}_{\mathrm{s}}(t)-\mathbf{v}_{\text {tri }}(t)\right] \cdot \mathbf{n}_{\text {tri }}<0$, where $\mathbf{v}_{\mathrm{s}}(t)$ and $\mathbf{v}_{\text {tri }}(t)$ are the velocities of the solvent particle and of the center of mass of the membrane triangle, respectively, and $\mathbf{n}_{\text {tri }}$ is the normal vector of the triangle, which is oriented toward the outside (inside) for external (internal) particles.

With the algorithm described above, it is very difficult to investigate the limit of vanishing membrane viscosity $\eta_{\mathrm{mb}}$ $=0$, because $\eta_{\mathrm{mb}}$ has a lower bound for the bond-flip procedure of a dynamically triangulated surface (compare Fig. 1). Thus, we employ a different solvent-membrane interaction for the case $\eta_{\mathrm{mb}}=0$. Instead of bounce-back collisions, the solvent particles are scattered elastically from the membrane triangles, and the vertices are not included in the MPCD collision procedure. In this way, only normal forces act on the membrane; no tangential stress is transferred. The membrane can be considered as a mathematical surface, which separates the inside of the vesicle from the outside, but has no tangential motion. The boundary condition of zero slip between inside and outside fluid still holds, because the collision boxes in the MPCD collision step are large enough to strongly couple the two fluids close to the membrane.

The number of solvent particles, which is placed inside the vesicle initially, determines an average volume $V$. This can be much smaller than the volume of a sphere of equal area, if the number of inside solvent particles is sufficiently small. However, since the MPCD fluid is compressible, the volume $V$ is not constant, but changes with bending rigidity or shear forces. Thus, the volume constraint introduced in $\mathrm{Sec}$. II C is necessary to control the volume to high accuracy.

\section{Parameters}

The parameters of the mesoscopic solvent model have already been specified in Sec. II A above.

We use the time steps $\Delta t_{\mathrm{CD}}=\Delta t_{\mathrm{BF}}=20 \Delta t_{\mathrm{MD}}$ and $\Delta t_{\mathrm{BS}}$ $=5 \Delta t_{\mathrm{MD}} \cdot \Delta t_{\mathrm{CD}}$ and $\Delta t_{\mathrm{BF}}$ should be larger than $\Delta t_{\mathrm{BS}}$ and $10 \Delta t_{\mathrm{MD}}$, respectively, to obtain thermal fluctuations correctly.

The average penetration of the solvent particles into the membrane film can easily be calculated from the MaxwellBoltzmann distribution of velocities. The result is very well approximated by $0.6 \lambda \Delta t_{\mathrm{BS}} / \Delta t_{\mathrm{CD}}$. With the parameters mentioned above, the typical penetration depth is $0.005 a$.

We used $l_{\mathrm{BS}}=0.05 a$. The vesicle dynamics is not sensitive to the scattering distance $l_{\mathrm{BS}}$, as long as $l_{\mathrm{BS}} \ll a$. We have investigated $l_{\mathrm{BS}} / a=0.006,0.05,0.1$, and 0.15 , and checked that the $l_{\mathrm{BS}}$ dependence is less than the statistical error.

The results are conveniently expressed in terms of dimensionless variables, the reduced volume $V^{*}=V /\left(4 \pi R_{0}^{3} / 3\right)$, the intrinsic time scale $\tau=\eta_{0} R_{0}^{3} / \kappa$, the reduced shear rate $\dot{\gamma}^{*}$ $=\dot{\gamma} \tau$, and the relative membrane viscosity $\eta_{\mathrm{mb}}^{*}=\eta_{\mathrm{mb}} / \eta_{0} R_{0}$. The relative membrane viscosity can also be written as

$$
\begin{aligned}
\eta_{\mathrm{mb}}^{*} & =\left(\eta_{\mathrm{mb}} a / \sqrt{m_{\mathrm{mb}} k_{\mathrm{B}} T}\right) \sqrt{m_{\mathrm{mb}} / m_{\mathrm{s}}}\left(a / R_{0}\right) \sqrt{m_{\mathrm{s}} k_{\mathrm{B}} T} /\left(\eta_{0} a^{2}\right) \\
& =0.0277 \eta_{\mathrm{mb}} a / \sqrt{m_{\mathrm{mb}} k_{\mathrm{B}} T},
\end{aligned}
$$

which is helpful to calculate $\eta_{\mathrm{mb}}^{*}$ from the membrane viscosity shown in Fig. 1. The error bars of the data presented below are typically estimated from the standard deviations of three or four independent runs.

Our membrane only behaves as a viscous fluid if there is a sufficient number of bond flips, which allow a relaxation of mechanical stresses. As a rough estimate, we require that there be at least one flip per bond within an average tanktreading period, which is larger than $2 \pi \tau$ in our simulations, so that $\Delta t_{\mathrm{BF}} / \Gamma \psi \leq 2 \pi \tau$. With $\tau=\eta_{0} R_{0}^{3} / \kappa$ and expression (3) for $\eta_{0}$ in the collisional regime $\lambda / a \ll 1$, we find

$$
2 \pi \Gamma \psi \frac{n-1}{18} \frac{k_{\mathrm{B}} T}{\kappa}\left(\frac{R_{0}}{a}\right)^{3}\left(\frac{a}{\lambda}\right)^{2} \gtrsim 1 .
$$

For $\lambda / a=0.025, R_{0} / a=5.68$, a bond-flip acceptance rate $\Gamma$ $=10^{-2}$, and $\kappa / k_{\mathrm{B}} T=20$, this implies $\psi \geq 0.002$, so that all runs are done in the viscous regime.

\section{E. Free energy of vesicle shapes}

For the description of the effect of shear flow on a vesicle on the basis of a simplified model, we need the free energy 


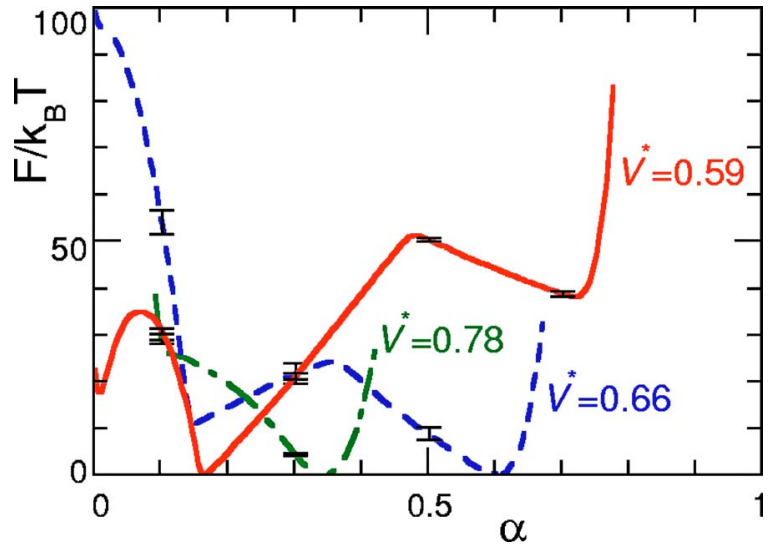

FIG. 2. (Color online) Free energy $F(\alpha)$ as a function of the asphericity $\alpha$ for the reduced volumes $V^{*}=0.59$ (solid line), 0.66 (dashed line), and 0.78 (dash-dotted line) in the absence of shear flow. Error bars are shown for a few data points.

of vesicles in thermal equilibrium. Therefore, we calculate the free energy $F$ using a version of the generalizedensemble Monte Carlo method (see Appendix A). Figure 2 shows the free energy as a function of the asphericity $\alpha$. The asphericity is the degree of deviation from a spherical shape [61],

$$
\alpha=\frac{\left(\lambda_{1}-\lambda_{2}\right)^{2}+\left(\lambda_{2}-\lambda_{3}\right)^{2}+\left(\lambda_{3}-\lambda_{1}\right)^{2}}{2 R_{\mathrm{g}}^{4}},
$$

where $\lambda_{1} \leqslant \lambda_{2} \leqslant \lambda_{3}$ are the eigenvalues of the moment-ofinertia tensor (with corresponding eigenvectors $\mathbf{u}_{1}, \mathbf{u}_{2}$, and $\mathbf{u}_{3}$ ) and the squared radius of gyration $R_{\mathrm{g}}^{2}=\lambda_{1}+\lambda_{2}+\lambda_{3}$. This is a convenient measure to distinguish oblate and prolate shapes, where $\alpha=0$ for spheres, 1 for thin rods, and 0.25 for thin disks [62]. Figure 2 shows that at reduced volume $V^{*}$ $=0.78$, only prolate shapes are stable; no metastable shapes exist. At $V^{*}=0.66$, in addition to the stable prolate shape, a biconcave discocyte shape appears as a metastable state. At $V^{*}=0.59$, the biconcave discocyte shape is stable, and prolate and stomatocyte shapes are metastable. A list of stable and metastable shapes for the various reduced volumes is given in Table I.

These stabilities agree well with previous studies $[2,63]$ based on the minimization of the curvature energy. The studies show that the lowest-energy state is a stomatocyte for $V^{*} \lesssim 0.59$, a discocyte for $0.59 \leqq V^{*} \lesssim 0.65$, and a prolate shape for $V^{*} \gtrsim 0.65$ [63]. Our simulation method has the advantage that it gives information not only about the minima of the free energy, but as well about the transition states

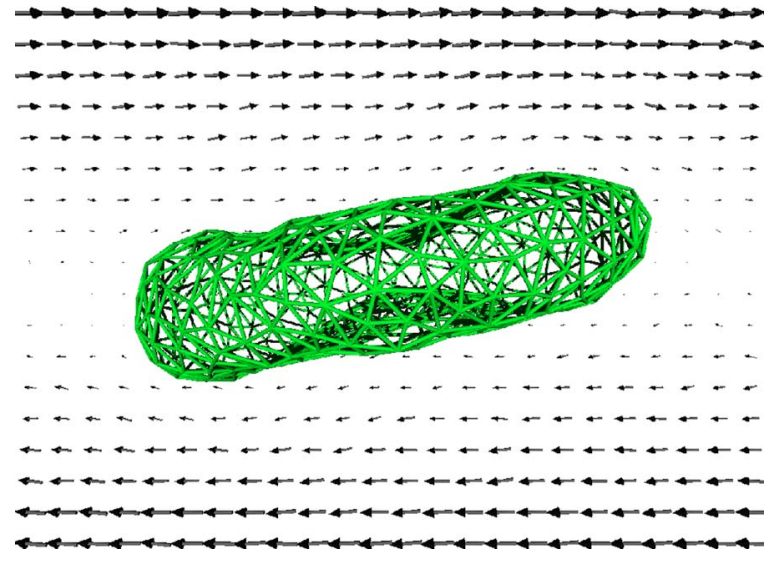

FIG. 3. (Color online) Snapshot of a discocyte vesicle under simple shear flow at the reduced shear rate $\dot{\gamma}^{*}=0.92$ and $\eta_{\mathrm{mb}}^{*}=0$. The arrows represent the velocity field in the $x z$ plane.

between these minima. Furthermore, it properly takes into account thermal fluctuations, which for a bending rigidity of $\kappa=20 k_{\mathrm{B}} T$ have a small effect at the free-energy minima, but are important near the saddle points.

\section{SIMULATION RESULTS}

\section{A. Vesicle shapes in shear flow}

At membrane viscosity $\eta_{\mathrm{mb}}^{*}=0$, a vesicle exhibits tanktreading motion for all simulated reduced volumes in the range $0.59 \leqslant V^{*} \leqslant 0.96$. A tank-treading vesicle has a finite inclination angle $\theta$ with the flow direction as shown in Figs. 3 and 4 . The inclination angle is given by $\theta=\arctan \left(u_{3}^{x} / u_{3}^{z}\right)$, where $\mathbf{u}_{3}=\left(u_{3}^{x}, u_{3}^{y}, u_{3}^{z}\right)$ is the eigenvector of the moments-ofinertia tensor with the largest eigenvalue. Figure 4 shows average angles $\langle\theta\rangle$ and tank-treading angular velocities $\langle\omega\rangle$ at $\eta_{\mathrm{mb}}^{*}=0$. These angles and angular velocities agree very well with the predictions of the theory of Keller and Skalak (KS). The KS theory is based on the approximations of a fixed ellipsoidal shape and a simple velocity field on the surface $[16,18]$ (see Appendix B). A similarly good agreement with the KS theory was obtained in the simulation of vesicles by the boundary integral method [20]. KS theory therefore provides a very good quantitative description of inclination angles and tank-treading frequencies at $\eta_{\mathrm{mb}}^{*}=0$.

The dependence of the inclination angle $\langle\theta\rangle$ on the size of the simulation box is discussed in Appendix $\mathrm{C}$. We find that for our standard simulation box, finite-size effects are sufficiently small to be less than or on the order of the statistical accuracy.

TABLE I. Stable and metastable shapes of vesicles, with the shape transitions induced by shear flow as obtained in the simulations.

\begin{tabular}{lll}
\hline \hline Reduced volume $V^{*}$ & Shapes without shear & Shape transitions in shear flow \\
\hline $0.78,0.91,0.96$ & Prolate & None \\
0.66 & Prolate, discocyte & discocyte to prolate, shape oscillations \\
0.59 & discocyte, stomatocyte, prolate & $\begin{array}{l}\text { discocyte to prolate, stomatocyte to prolate, prolate to } \\
\text { discocyte, shape oscillations between prolate and discocyte }\end{array}$ \\
\hline \hline
\end{tabular}




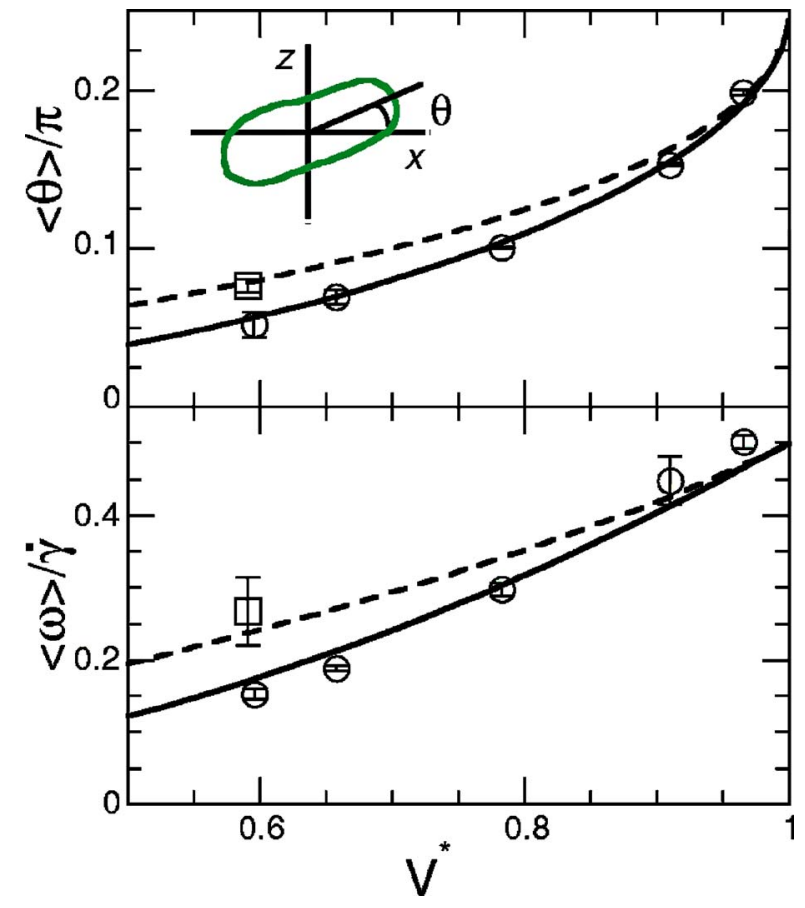

FIG. 4. (Color online) Dependence of the average inclination angle $\langle\theta\rangle(-\pi / 2 \leqslant \theta<\pi / 2)$ and average angular velocity $\langle\omega\rangle$ on the reduced volume $V^{*}$ at the reduced shear rate $\dot{\gamma}^{*}=0.92$ and $\eta_{\mathrm{mb}}^{*}=0$. Circles and squares represent prolate and discocyte vesicles, respectively. The solid and dashed lines are calculated by KS theory for prolate and oblate ellipsoids, respectively.

With increasing membrane viscosity $\eta_{\mathrm{mb}}^{*}$, the inclination angle $\langle\theta\rangle$ decreases, as shown in Fig. 5. The qualitative features of the simulation data are reproduced quite well by the KS theory. In the KS theory, the vesicle transits from tanktreading to tumbling motion when the angle $\theta$ reaches zero. In contrast, we find that for small reduced volumes, the vesicle starts to tumble intermittently already for nonzero $\langle\theta\rangle$. An example of tumbling motion at $V^{*}=0.78$ is shown in Fig. 6. The origins of this occasional tumbling motion are thermal fluctuations of the orientation of the long axis of the vesicle, which are included in our simulations. Figure 7 shows the critical membrane viscosity $\eta_{\mathrm{mb}}^{c}$, where intermittent tumbling is first observed. This intermittent tumbling motion smoothes out the decrease in $\langle\theta\rangle$ around the transition point (compare Fig. 5). At larger $\eta_{\mathrm{mb}}^{*}$, tumbling becomes more periodic.

Deviations from the KS predictions are also observed for large reduced volumes. For quasispherical vesicles, with reduced volumes $V^{*}=0.91$ and 0.96 , the inclination angle $\langle\theta\rangle$ decreases more slowly than predicted by KS theory (see Fig. 5 ), although the tank-treading frequency $\langle\omega\rangle$ shows only slight deviations (compare Fig. 8). We believe that the physical origin of the deviations in the inclination angle in this case is shape fluctuations of the sheared vesicle, since the eigenvectors of the moment-of-inertia tensor of a quasispherical vesicle can change their direction without a membrane rotation. For example, a fluctuation that elongates the vesicle in the $\mathbf{u}_{2}$ direction and shrinks it in the $\mathbf{u}_{3}$ direction corresponds to a discrete $\pi / 2$ jump of the inclination angle
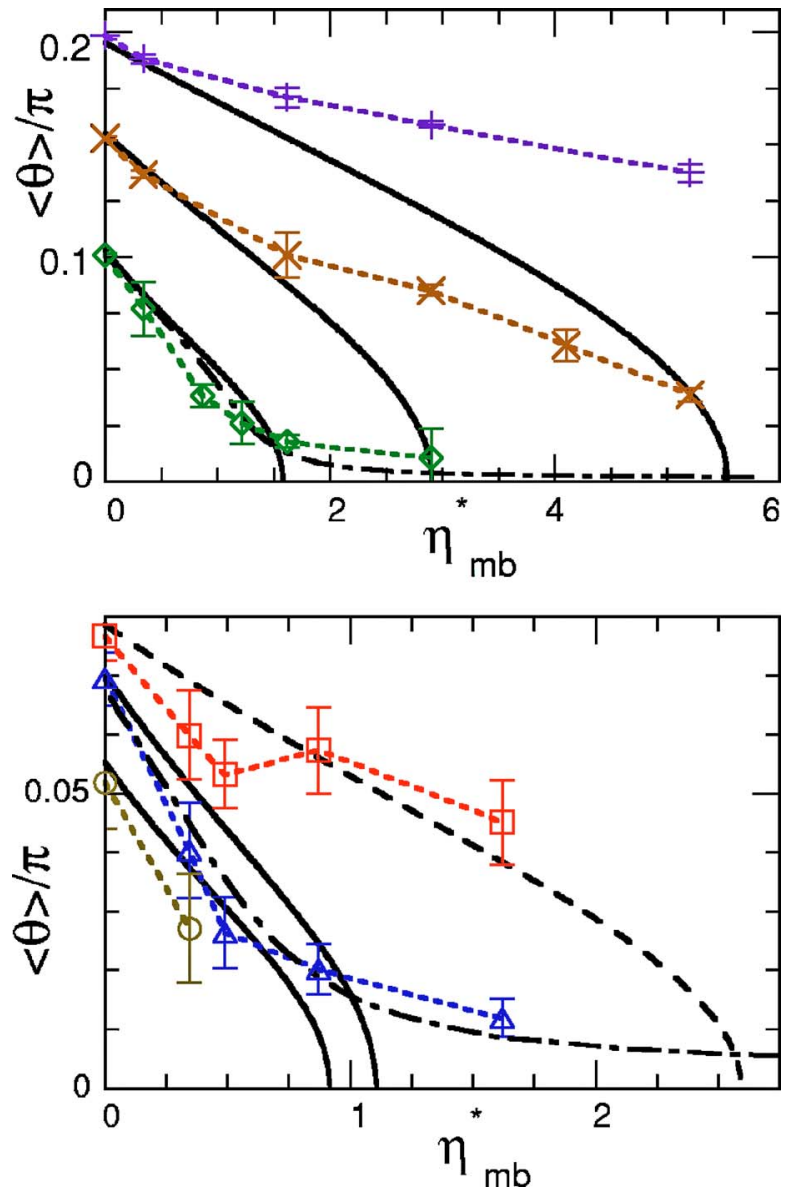

FIG. 5. (Color online) Dependence of the average inclination angle $\langle\theta\rangle$ on the membrane viscosity $\eta_{\mathrm{mb}}^{*}$ for $\dot{\gamma}^{*}=0.92$ and various volumes $V^{*}$. Circles and squares represent prolate and discocyte vesicles at $V^{*}=0.59$, respectively. Triangles, diamonds, crosses, and pluses represent vesicles at $V^{*}=0.66,0.78,0.91$, and 0.96 . The solid and dashed lines are calculated by KS theory for prolate $\left(V^{*}\right.$ $=0.59,0.66,0.78,0.91$, and 0.96$)$ and oblate ellipsoids $\left(V^{*}=0.59\right)$, respectively. The dash-dotted lines are calculated by KS theory and Eq. (19) with rotational Péclet number $\chi=600$, for $V^{*}=0.66$ and 0.78 .

at $\lambda_{2}=\lambda_{3}$. In shear flow, the shrinkage of the long axis during tumbling can lead to a $\theta$ rotation in the opposite direction of tank treading (see tumbling at $t / \tau=75$ in Fig. 6). This occurs more frequently at larger $V^{*}$; for $V^{*}=0.78$, the long axis jumps to a different orientation only in about $10 \%$ of tumbling events, while this is the case in $90 \%$ of (attempted) turns for $V^{*}=0.91$ (at $\dot{\gamma}^{*}=0.92$ ). Since this motion of the shape deformation is normal to the membrane surface, it should be little affected by the membrane viscosity $\eta_{\mathrm{mb}}^{*}$, and thus reduce the $\eta_{\mathrm{mb}}^{*}$ dependence of $\langle\theta\rangle$.

Our two different models for membranes with vanishing and nonvanishing membrane viscosity $\eta_{\mathrm{mb}}$ have been described in Sec. II C above. It is very nice to see now that these two models give consistent results; the data for $\langle\theta\rangle$ and $\langle\omega\rangle$ at $\eta_{\mathrm{mb}}=0$ agree very well with the extrapolation of the results for nonslip boundary conditions on the membrane with $\eta_{\mathrm{mb}}>0$ (compare Figs. 5 and 8). 


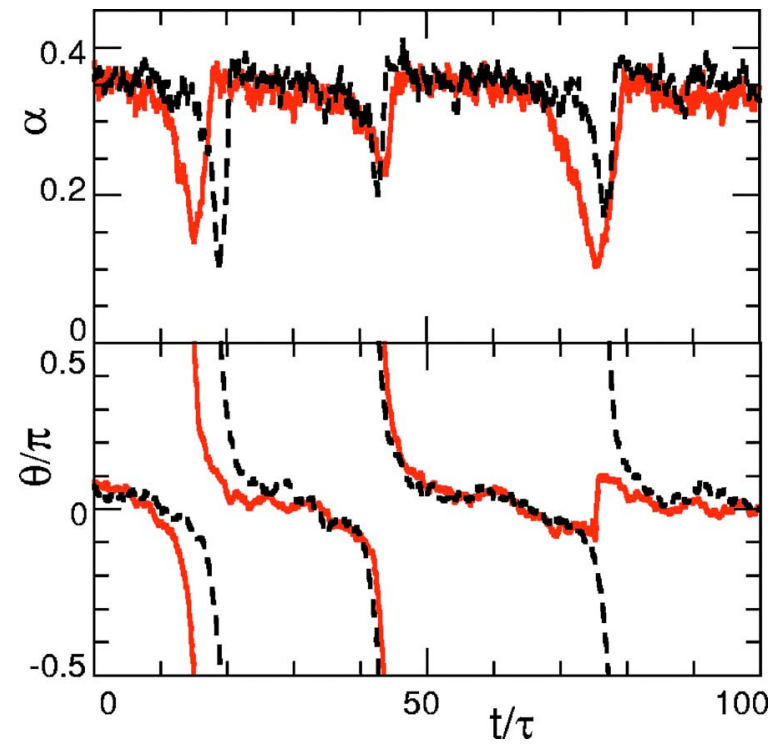

FIG. 6. (Color online) Time dependence of asphericity $\alpha$ and inclination angle $\theta$, for $V^{*}=0.78, \eta_{\mathrm{mb}}^{*}=1.62$, and $\dot{\gamma}^{*}=0.92$. The dashed lines are obtained from Eqs. (18) and (19) with $\zeta_{\alpha}^{*}=100$, $\zeta_{\theta}^{*}=30, A^{*}=18$, and $B(\alpha)=1.0$.

\section{B. Shear-induced shape transformation}

As a function of shear rate, reduced volume, and membrane viscosity, we observe several types of phase transformation. We have studied two reduced volumes in detail, $V^{*}=0.59$, where the free-energy function of a vesicle has three minima, and $V^{*}=0.66$, where two minima exist (compare Fig. 2). The results are summarized in Table I and the phase diagram of Fig. 9.

For reduced volume $V^{*}=0.59$, the discocyte is stable in the absence of shear flow. In our simulations with membrane viscosity $\eta_{\mathrm{mb}}^{*}=0$, we find that the discocyte is destabilized and transforms into a prolate shape at shear rates $\dot{\gamma}^{*} \geqslant 1.66$. However, for a smaller shear rate of $\dot{\gamma}^{*}=0.92$, the discocyte vesicle retains its shape. At an intermediate shear rate of $\dot{\gamma}^{*}=1.38$, some discocytes transform into prolate shapes, while others keep their original shape.

The inclination angle $\langle\theta\rangle$ of prolates decreases faster than that of discocytes with increasing $\eta_{\mathrm{mb}}^{*}$, as shown in Fig. 5 for

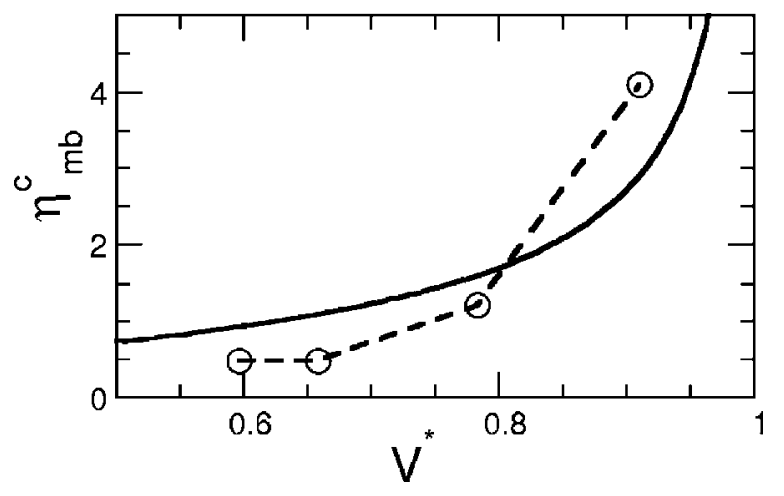

FIG. 7. Relative membrane viscosity $\eta_{\mathrm{mb}}^{\mathrm{c}}$, at which tumbling motion is first observed for $\dot{\gamma}^{*}=0.92$. The simulation data $(\bigcirc)$ are compared with the results of KS theory (solid line) for prolate shapes.

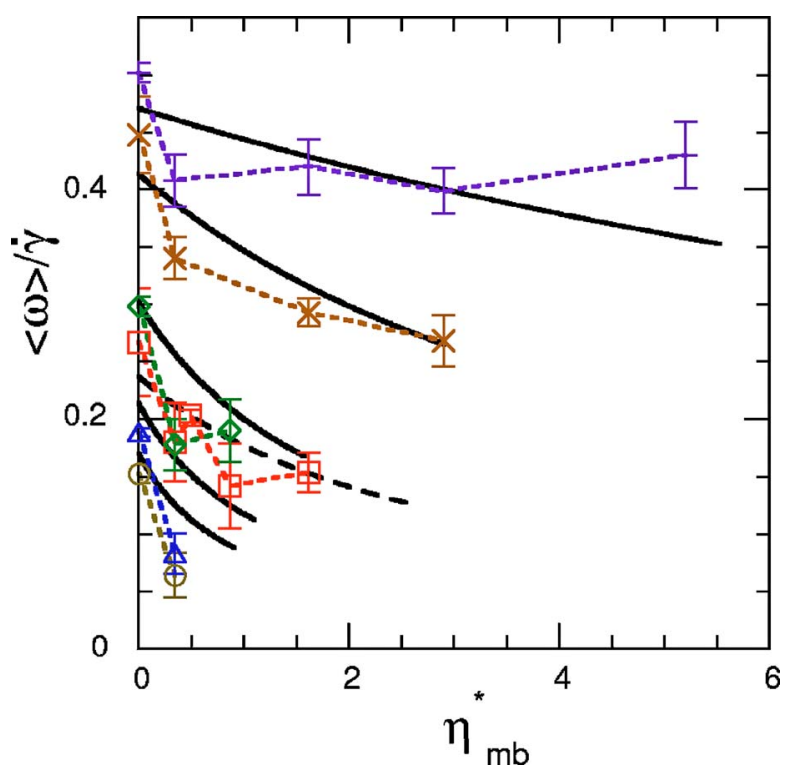

FIG. 8. (Color online) Dependence of the average angular velocity $\langle\omega\rangle$ on the membrane viscosity $\eta_{\mathrm{mb}}^{*}$ for shear rate $\dot{\gamma}^{*}=0.92$ and various volumes $V^{*}$. Circles and squares represent prolate and discocyte vesicles at $V^{*}=0.59$, respectively. Triangles, diamonds, crosses, and pluses represent vesicles at $V^{*}=0.66,0.78,0.91$, and 0.96 . The solid and dashed lines are calculated by KS theory for prolate $\left(V^{*}=0.59,0.66,0.78,0.91\right.$, and 0.96$)$ and oblate ellipsoids $\left(V^{*}=0.59\right)$, respectively.

$\dot{\gamma}^{*}=0.92$. For membrane viscosities $\eta_{\mathrm{mb}}^{*} \geqslant 0.49$, the prolate shape enters the tumbling phase (compare Fig. 7), while the discocyte remains in the tank-treading state. The reason is that the discocyte has a flat dimple region and is less affected by the membrane viscosity than the prolate shape. In this regime, the tank-treading prolate starts tumbling intermittently and then transforms into a discocyte, as shown in Fig.

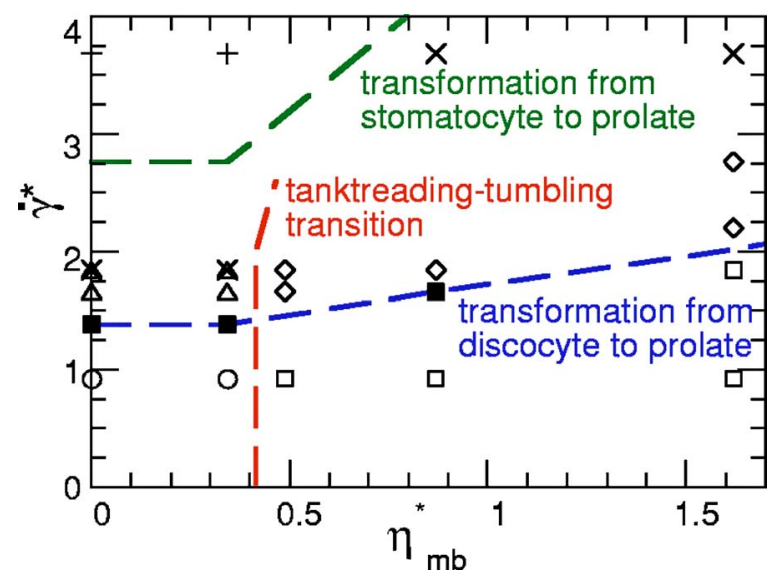

FIG. 9. (Color online) Dynamical phase diagram of a vesicle in shear flow, for reduced volume $V^{*}=0.59$. Symbols show simulated parameter values, and indicate tank-treading discocyte and tanktreading prolate $(\bigcirc)$, tank-treading prolate and unstable discocyte $(\triangle)$, tank-treading discocyte and tumbling (transient) prolate $(\square)$, tumbling with shape oscillation $(\diamond)$, unstable stomatocyte $(+)$, stable stomatocyte $(\times)$, and near transition $(\mathbf{\square})$. The dashed lines are guides to the eye. 


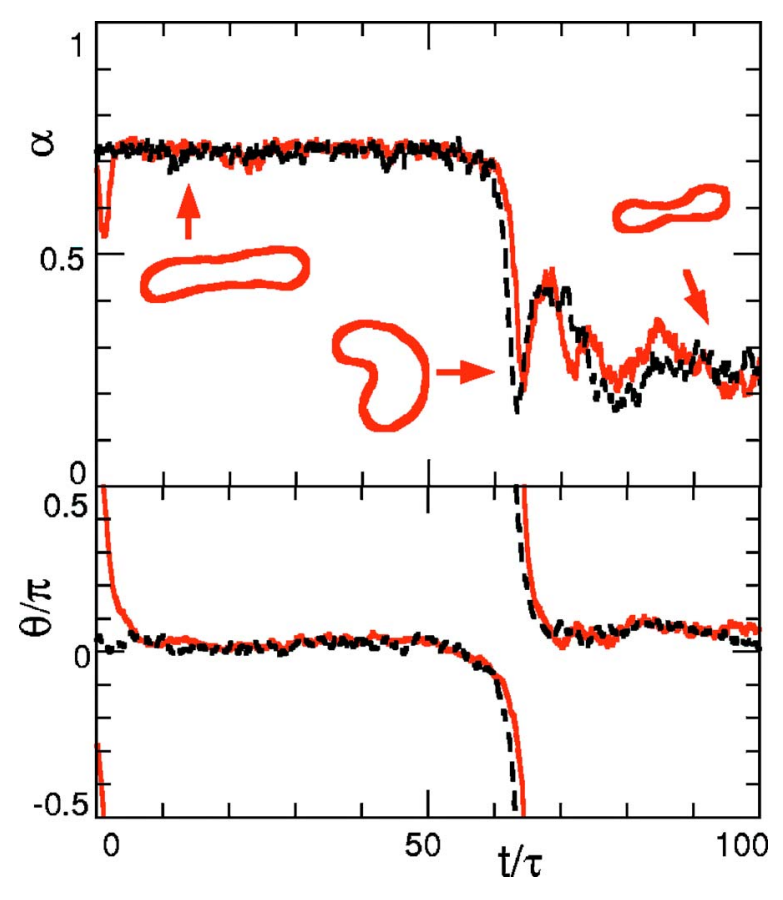

FIG. 10. (Color online) Time dependence of asphericity $\alpha$ and inclination angle $\theta$, for $V^{*}=0.59, \eta_{\mathrm{mb}}^{*}=0.87$. The dashed lines are obtained from Eqs. (18) and (19) with $B(\alpha)=1.12-0.14 \alpha$. The other parameters are set to the same values in Fig. 6. Snapshots of the vesicle cross section in the $x z$ plane are also shown.

10 for $\eta_{\mathrm{mb}}^{*}=0.87$. At membrane viscosity $\eta_{\mathrm{mb}}^{*}=0.87$, the discocyte is a stable tank-treading state for $\dot{\gamma}^{*} \lesssim 1.7$. For larger shear rates, however, the discocyte transforms into a prolate shape and the vesicle exhibits tumbling accompanied by shape oscillations between discocyte and prolate. For $\dot{\gamma}^{*}$ $=0.92$, the lifetime of the metastable tank-treading prolate is $(70 \pm 40) \tau$ and $(40 \pm 20) \tau$ at $\eta_{\mathrm{mb}}^{*}=0.49$ and 0.87 , respectively. At a larger membrane viscosity $\eta_{\mathrm{mb}}^{*}=1.62$, the tank-treading discocyte is stable even at $\dot{\gamma}^{*}=1.84$ [37].

The transformation of a stomatocyte vesicle requires much higher shear rates than for a discocyte. Figure 11 shows the dynamics of stomatocyte with $V^{*}=0.59$ and $\eta_{\mathrm{mb}}^{*}$ $=0.34$. A shear flow with $\dot{\gamma}^{*}=1.84$ elongates the stomatocyte into an ellipsoidal shape, but the vesicle retains its inside bud. At a higher shear $\dot{\gamma}^{*}=3.68$, the stomatocyte is found to transform into a prolate shape, for both $\eta_{\mathrm{mb}}^{*}=0$ and $\eta_{\mathrm{mb}}^{*}$ $=0.34$. The snapshot in Fig. 11 captures the vesicle in a state where the inside is just pulled open. It is interesting to note that in the stomatocyte state, the neck is preferentially oriented in the vorticity direction (normal to the flow and gradient directions). This orientation is clearly favorable in order to minimize dissipation in the internal fluid, because in this case the bud position is stationary. At high membrane viscosity $\eta_{\mathrm{mb}}^{*}=0.87$ and 1.62 , the stomatocyte retains its shape even for $\dot{\gamma}^{*}=3.68$. Thus, a high membrane viscosity also stabilizes stomatocytes under shear.

For reduced volume $V^{*}=0.66$, the discocyte is a metastable state in the absence of shear flow. The threshold in the shear rate to induce the transformation into a prolate shape is $\dot{\gamma}^{*} \simeq 0.2$ at $\eta_{\mathrm{mb}}^{*}=0$. This is a much smaller shear rate than for $V^{*}=0.59$. At $\eta_{\mathrm{mb}}^{*} \geqslant 0.49$, prolate vesicles enter the tumbling

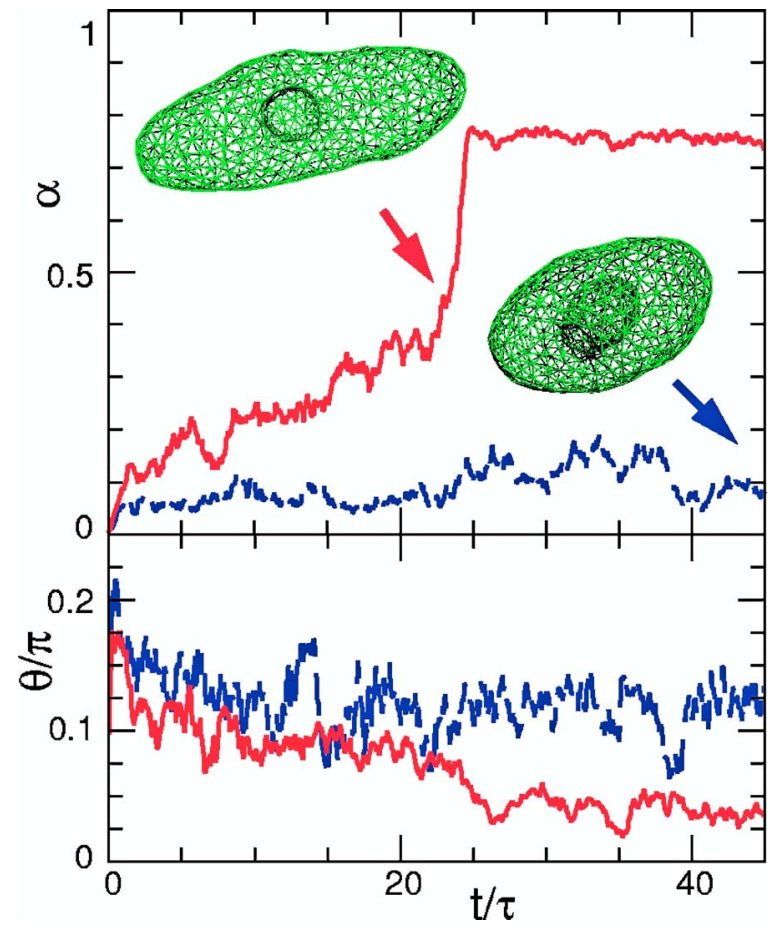

FIG. 11. (Color online) Time dependence of asphericity $\alpha$ and inclination angle $\theta$, for $V^{*}=0.59$ and $\eta_{\mathrm{mb}}^{*}=0.34$. The dashed and solid lines represent the vesicle at $\dot{\gamma}^{*}=1.84$ and 3.68 , respectively. Snapshots of vesicles (a tank-treading stomatocyte at $t / \tau=45$, and a vesicle during the stomatocyte-to-prolate transformation at $t / \tau=23$ ) are also shown.

phase, see Fig. 7. In contrast to the case of $V^{*}=0.59$, the vesicle continues its tumbling motion with shape oscillations between prolate and discocyte shapes even at a small shear rate of $\dot{\gamma}^{*}=0.92$, since there is no metastable tank-treading discocyte for $V^{*}=0.66$.

Thus, we find that the threshold in the shear rate for the discocyte-to-prolate transformation depends on the reduced volume $V^{*}$. In a previous study [20] of vesicles in shear flow with the boundary integral method, tank-treading prolates were found as the only stable shape for all investigated shear rates $\dot{\gamma}^{*} \geqslant 1.0$ and all reduced volumes $V^{*} \geqslant 0.52$; a tanktreading discocyte was not observed. We believe that the results of Ref. [20] and our simulations are not inconsistent, and predict that calculations with the boundary-integral method should also yield stable discocytes for shear rates smaller than $\dot{\gamma}^{*}=0.1 \ldots 1$.

\section{SIMPLIFIED STOCHASTIC MODEL}

\section{A. The model}

The theory of Keller and Skalak [16] and Tran-Son-Tay et al. [18] qualitatively explains the $\eta_{\mathrm{mb}}^{*}$ dependence of the stability of tank-treading (compare Fig. 5). However, KS theory cannot be employed to describe morphological changes, and does not take into account thermal fluctuations. Therefore, we suggest a simplified phenomenological model, which is defined by the stochastic equations 


$$
\begin{gathered}
\zeta_{\alpha} \frac{d}{d t} \alpha=-\partial F / \partial \alpha+A \dot{\gamma} \sin (2 \theta)+\zeta_{\alpha} g_{\alpha}(t), \\
\frac{d}{d t} \theta=\frac{1}{2} \dot{\gamma}\{-1+B(\alpha) \cos (2 \theta)\}+g_{\theta}(t),
\end{gathered}
$$

with Gaussian white noises $g_{\alpha}$ and $g_{\theta}$, which are determined by

$$
\begin{aligned}
\left\langle g_{\alpha}(t)\right\rangle=\left\langle g_{\theta}(t)\right\rangle & =\left\langle g_{\alpha}(t) g_{\theta}\left(t^{\prime}\right)\right\rangle=0, \\
\left\langle g_{\alpha}(t) g_{\alpha}\left(t^{\prime}\right)\right\rangle & =2 D_{\alpha} \delta\left(t-t^{\prime}\right), \\
\left\langle g_{\theta}(t) g_{\theta}\left(t^{\prime}\right)\right\rangle & =2 D_{\theta} \delta\left(t-t^{\prime}\right),
\end{aligned}
$$

friction coefficients $\zeta_{\alpha}$ and $\zeta_{\theta}$, and diffusion constants $D_{\alpha}$ $=k_{\mathrm{B}} T / \zeta_{\alpha}$ and $D_{\theta}=k_{\mathrm{B}} T / \zeta_{\theta}$. Note that $\zeta_{\theta}$ does not appear in Eq. (15); it drops out because the shear force is also caused by friction.

The form of the stochastic equations (14) and (15) is motivated by the following considerations. The first term in Eq. (14), $\partial F / \partial \alpha$, is the thermodynamic force due to bending energy and volume constraints; it is calculated from the free energy $F(\alpha)$ of Fig. 2. The second term of Eq. (14) is the deformation force due to the shear flow. Its $\theta$ dependence can be deduced from the shape equations of Ref. [20]. Those shape equations are obtained under the assumption of mirror symmetry of vesicle shape with respect to a plane defined by the long axis of the vesicle, and imply that the hydrodynamic force which induces local membrane deformation is proportional to $\sin (2 \theta)$. Since our model contains only global variables of vesicle shape, the force on $\alpha$ in principle has to be found by integrating the local force over the membrane surface. While this cannot be done explicitly, it implies that the force on $\alpha$ should depend linearly on $\sin (2 \theta)$. The amplitude $A$ is assumed to be independent of the asphericity $\alpha$ to leading order. Equation (15) is adapted from Eq. (B3) of KS theory (see Appendix B). In KS theory, $B$ is a constant which depends on the ratio $\eta_{\text {in }} / \eta_{0}$ of internal and external viscosities, the membrane viscosity $\eta_{\mathrm{mb}}$, and the ellipsoid shape. In general, $B$ decreases with increasing $\eta_{\text {in }} / \eta_{0}$ as well as $\eta_{\mathrm{mb}}$ [compare Eq. (B4)], and also with increasing asphericity. For $B>1$, tank-treading motion occurs, while for $B<1$, tumbling motion is predicted.

We introduce dimensionless variables

$$
\begin{gathered}
\zeta_{\alpha}^{*}=\frac{\zeta_{\alpha}}{\kappa \tau}, \quad \zeta_{\theta}^{*}=\frac{\zeta_{\theta}}{\kappa \tau}, \quad A^{*}=\frac{A}{\kappa \tau}, \quad \tilde{t}=t / \tau, \\
D_{\alpha}^{*}=\frac{k_{\mathrm{B}} T}{\kappa \zeta_{\alpha}^{*}}=D_{\alpha} \tau, \quad D_{\theta}^{*}=\frac{k_{\mathrm{B}} T}{\kappa \zeta_{\theta}^{*}}=D_{\theta} \tau .
\end{gathered}
$$

This implies

$$
\zeta_{\alpha}^{*} \dot{\alpha}=-\kappa^{-1} \partial F / \partial \alpha+A^{*} \dot{\gamma}^{*} \sin (2 \theta)+\zeta_{\alpha}^{*} \widetilde{g}_{\alpha}(\widetilde{t}),
$$

$$
\dot{\theta}=\frac{1}{2} \dot{\gamma}^{*}\{-1+B(\alpha) \cos (2 \theta)\}+\widetilde{g}_{\theta}(\widetilde{t}),
$$

where $\dot{\alpha} \equiv d \alpha / d \widetilde{t}$, and the rescaled Gaussian white noises satisfy

$$
\begin{aligned}
& \left\langle\widetilde{g}_{\alpha}(\widetilde{t}) \widetilde{g}_{\alpha}\left(\vec{t}^{\prime}\right)\right\rangle=2 D_{\alpha}^{*} \delta\left(\widetilde{t}-\widetilde{t}^{\prime}\right), \\
& \left\langle\widetilde{g}_{\theta}(\widetilde{t}) \widetilde{g}_{\theta}\left(\widetilde{t}^{\prime}\right)\right\rangle=2 D_{\theta}^{*} \delta\left(\widetilde{t}-\widetilde{t}^{\prime}\right) .
\end{aligned}
$$

\section{B. Shape transitions}

For prolate vesicles with reduced volume $V^{*}=0.78$, whose shape changes little during tumbling ( $\alpha \simeq 0.34$; compare Fig. 2 ), the dynamics is reproduced very well with constant $B$ $=1$ (see Fig. 6). At $V^{*}=0.59$ and 0.66 , however, the vesicle shape can vary strongly, so that the dependence of $B$ on the asphericity $\alpha$ has to be taken into account. For simplicity, we assume a linear relation $B(\alpha)=B_{0}-B_{1} \alpha$. To obtain tanktreading discocytes and tumbling prolate shapes, the conditions $B(0.2)>1$ and $B(0.7)<1$ are needed in the absence of stochastic forces. With the deterministic version of Eqs. (18) and (19), we were able to reproduced the vesicle dynamics at $V^{*}=0.59$ and $\eta_{\mathrm{mb}}^{*}=1.62$ very well (compare Fig. 4 in Ref. [37]). However, the deterministic version cannot describe the finite lifetime of tank-treading prolates (see Fig. 10).

For the stochastic equations (18) and (19), tumbling can already occur for $B$ slightly larger than unity, because the actual inclination angle can become negative by thermal fluctuations. This is the situation shown in Fig. 10, where the prolate vesicle is in the tank-treading state for a while, before it starts tumbling and transforms into a discocyte. Furthermore, the results of the stochastic model reproduce the simulated vesicle dynamics better than the deterministic version (compare Figs. 6 and 10). Thus, the intermittent tumbling is caused by thermal fluctuation.

In the absence of thermal fluctuations, both transitions (from a tank-treading to a tumbling prolate shape, and from a tank-treading discocyte to a tank-treading prolate shape) are saddle-node bifurcations. The vesicle changes its behavior when the stable fixed point in $(\alpha, \theta)$ space, which describes a tank-treading state, is annihilated by the unstable fixed point $[21,64]$. In the transition from tank-treading to tumbling prolate shape, these fixed points are located at $\theta$ $= \pm 0.5 \arccos (1 / B)$. In the transition from a tank-treading discocyte to tank-treading prolate shape, the fixed points correspond to the free-energy minimum of the discocyte, and the free-energy barrier between discocyte and prolate shapes. In the case of tumbling, no stable fixed point exists and limit cycle occurs. Thermal fluctuations change both transitions into continuous crossovers.

Our results show that the dynamical behavior in various parts of the phase diagram, Fig. 9, can be explained qualitatively on the basis of the phenomenological model (18) and (19). For a more quantitative comparison, analytical expressions for the parameters $\zeta_{\alpha}^{*}, A^{*}$, and $B(\alpha)$ are required. This is straightforward for $B(\alpha)$, which could be determined from KS theory, as explained in Appendix B. The difficult parts 


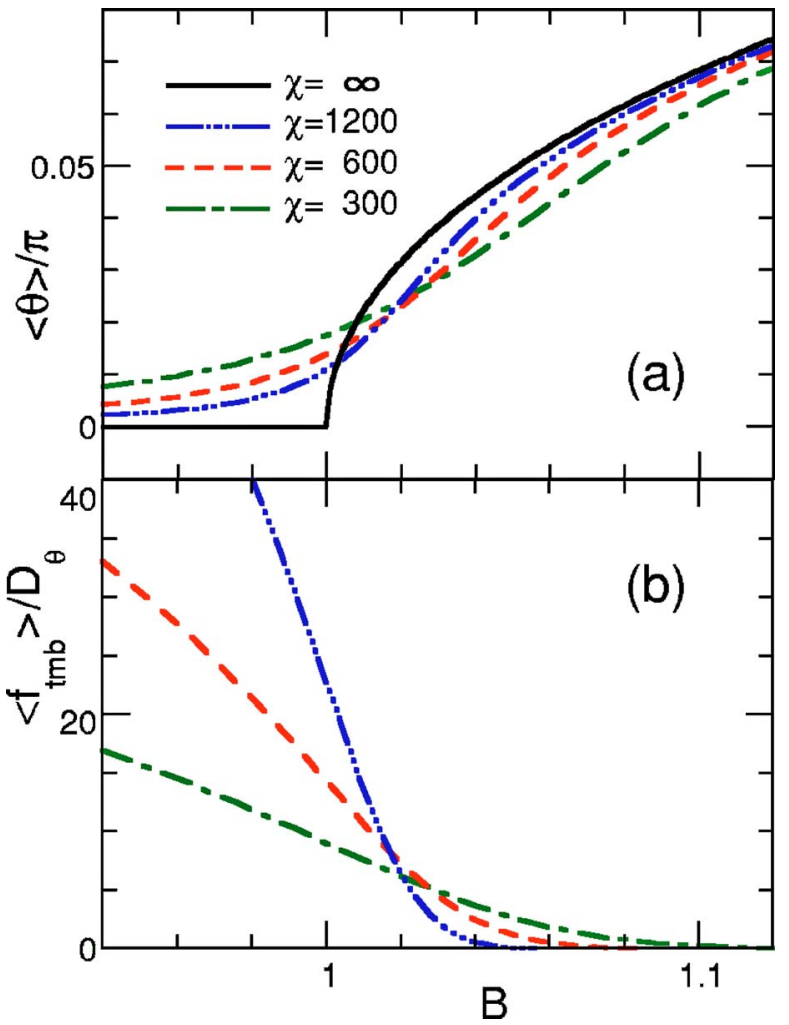

FIG. 12. (Color online) Dependence of (a) the mean angle $\langle\theta\rangle(-\pi / 2 \leqslant \theta<\pi / 2)$ and (b) the tumbling frequency $f_{\text {tmb }}$ on $B$ obtained from Eq. (19) with various relative shear rates $\chi$. The errors are less than (a) $0.0003 \pi$ and (b) 0.2 .

are $\zeta_{\alpha}^{*}$ and $A^{*}$, which in general depend on $\alpha$ and $\eta_{\mathrm{mb}}$. A single shape parameter $\alpha$ is sufficient to describe the shape transitions between discocyte and prolate shape. However, a single shape parameter is not always sufficient. For example, the transformation from stomatocyte to prolate does not progress through a discocyte shape in Fig. 11. Two shape parameters are necessary to describe this dynamics in a simplified model. Therefore, with our current phenomenological approach, the full phase diagram cannot be reproduced quantitatively.

\section{Importance of thermal fluctuations}

We want to discuss now the effect of thermal fluctuations in Eq. (19) with constant $B$, i.e., for fixed vesicle shapes. This corresponds to the original Keller-Skalak theory with thermal noise. The smoothing effect of thermal fluctuations on $\langle\theta\rangle$ around the tank-treading to tumbling transition, which we see in the simulations, is captured very well by the stochastic model (19), as demonstrated in Fig. 5 for $V^{*}=0.78$.

The importance of thermal fluctuations depends on the rotational Péclet number $\chi=\dot{\gamma} / D_{\theta}=\dot{\gamma} \zeta_{\theta} / k_{\mathrm{B}} T$. In terms of the dimensionless variables (17), the rotational Péclet number is given by $\chi=\dot{\gamma} / D_{\theta}=\dot{\gamma}^{*} / D_{\theta}^{*}=\dot{\gamma}^{*} \zeta_{\theta}^{*} \kappa / k_{\mathrm{B}} T$. The results are shown in Figs. 12 and 13. The steady probability distribution of $\theta$ only depends on $B$ and $\chi$. For $B<1$, the probability distribution of $\theta$ is symmetric for $\chi=\infty$. Thermal noise makes the distribution asymmetric and $\langle\theta\rangle>0$. For $B>1$, intermit-

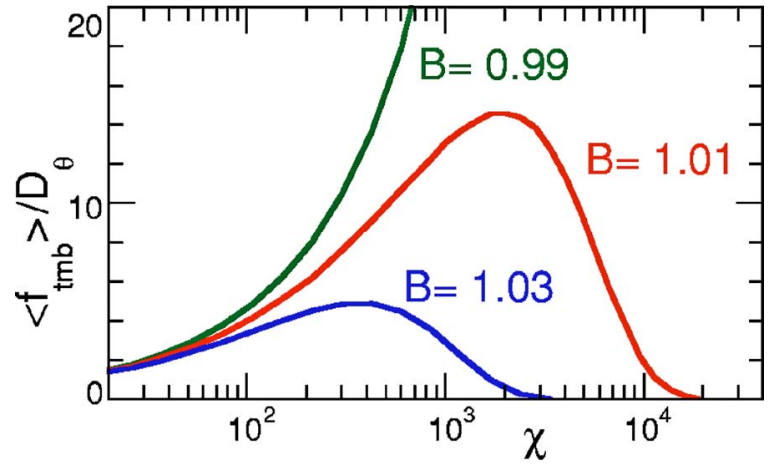

FIG. 13. (Color online) Dependence of the dimensionless tumbling frequency $\left\langle f_{\mathrm{tmb}}\right\rangle / D_{\theta}$ on the rotational Péclet number $\chi$, for different values of $B$ close to the transition point, as obtained from Eq. (19). The errors are less than 0.1.

tent tumbling decreases the value of $\langle\theta\rangle$. Furthermore, a lower relative shear rate $\chi$ induces more tumbling [see Figs. 12(b) and 13]. This result, which at first is very surprising, is due to the suppression of thermal fluctuations with increasing shear rate.

Kraus et al. [20] argue for $\eta_{\mathrm{mb}}^{*}=0$ that thermal fluctuations become important when the rotational Péclet number $\chi \lesssim 1$, i.e., for shear rates $\dot{\gamma} \tau \lesssim k_{\mathrm{B}} T / \kappa$, where the typical rotational diffusion time $\eta_{0} R_{0}^{3} / k_{\mathrm{B}} T$ becomes comparable to the inverse shear rate. This is different, however, for the tanktreading to tumbling transition. The effects of thermal fluctuations are most pronounced around the transition point $B$ $=1$. It can be seen in Fig. 12 that thermal fluctuations cannot be neglected for rotational Péclet numbers as large as $\chi$ $=1200$. We approximate the rotational friction coefficient by that of a sphere with radius $R_{0}$, which is given by $\zeta_{\theta}^{*}$ $=8 \pi \eta_{0} R_{0}^{3} / \kappa \tau=8 \pi \simeq 30$. This should be a reasonable approximation as long as the vesicle is not too elongated. It is a lower bound for elongated shapes, since $\zeta_{\theta}$ increases with the third power of the long axis for large ellipticity. In the quasispherical approximation, the relative shear rate is $\chi$ $\simeq 30 \dot{\gamma} \eta_{0} R_{0}^{3} / k_{\mathrm{B}} T$. Thus, $\chi=600$ corresponds to $\dot{\gamma} \tau=20 k_{\mathrm{B}} T / \kappa$.

A more detailed understanding of the tank-treading to tumbling transition can be obtained from Eq. (19). The force due to the flow field can be integrated to obtain a (dimensionless) effective potential for the motion of $\theta$, which is found to be

$$
V_{\mathrm{eff}}(\theta)=\frac{1}{2} \zeta_{\theta}^{*} \dot{\gamma}^{*}[\theta-0.5 B \sin (2 \theta)]
$$

Thus, the trapping energy for tank treading, i.e., the difference of the maximum and the minimum of the effective potential, is given by $0.5 \zeta_{\theta}^{*} \dot{\gamma}^{*}\left[-\arccos (1 / B)+\sqrt{B^{2}-1}\right]$. When the trapping energy is smaller than $k_{\mathrm{B}} T$, the vesicle escapes from the minimum (which describes the tank-treading state) and starts intermittent tumbling. As $B$ approaches unity, this trapping energy decreases as $(B-1)^{3 / 2}$, and intermittent tumbling can be induced at even larger values of $\chi$.

Note that we predict two different scenarios for a transition (or crossover) from tank treading to tumbling. 
(i) At constant shear rate, increasing membrane viscosity makes tank treading unstable and leads to a periodic tumbling state (compare Fig. 5).

(ii) For a fixed membrane viscosity, which is sufficiently small so that $B>1$, increasing shear rate (corresponding to increasing $\chi$ ) reduces the effect of rotational fluctuations, suppresses intermittent tumbling, and stabilizes the tanktreading state.

\section{Comparison with experiments}

A shear-induced transition from tumbling to tank treading with increasing shear rate has already been observed in experiments with red blood cells [13] and with liposomes [15]. However, KS theory cannot explain this transition. We propose that the suppression of thermal fluctuations with increasing shear rate is responsible for the observed behavior.

In the intermittent tumbling region, the tumbling frequency decreases and the lifetime of tank treading increases with increasing shear rate, as shown in Fig. 13. The transition from intermittent tumbling to tank treading occurs at $\chi$ $=10^{3}-10^{4}$. Although large vesicles are less affected by thermal fluctuations $\left(\chi \propto R_{0}^{3}\right)$, the thermal-fluctuation effect is significant even for micrometer-sized vesicles. Goldsmith and Marlow [13] reported that red blood cells in water (with $\left.\eta_{0}=0.001 \mathrm{~Pa} \mathrm{sec}\right)$ are tumbling in shear flow, while in $30 \%$ Dextran solution (with $\eta_{0}=0.05 \mathrm{~Pa} \mathrm{sec}$ ) a shear-induced transition from tumbling to tank treading occurs at $\dot{\gamma}$ $\simeq 1 \mathrm{sec}^{-1}$. They also observed an intermediate motion between tumbling and tank treading around the transition shear rate. We interpret this intermediate behavior as intermittent tumbling with a long lifetime of the tank-treading state. For their experimental values $\left(R_{0}=3 \mu \mathrm{m}, \eta_{0}=0.05 \mathrm{~Pa} s e c\right.$, and $\dot{\gamma}=1 \mathrm{sec}^{-1}$ ), we obtain a critical shear rate of $\chi^{\mathrm{c}}=10^{4}$. Thus, their observations are consistent with our results for $B$ $=1.01$.

\section{DISCUSSION}

A simple scaling argument shows that shear should affect the vesicle shape for shear rates $\dot{\gamma} \tau \gtrsim 1$, where the hydrodynamic forces on the vesicle shape $\left(\sim \dot{\gamma} \eta_{0} R_{0}^{3}\right)$ become similar to the force of vesicle curvature $(\sim \kappa)$. Since the critical shear rate for the shape transition is a factor $\kappa / k_{\mathrm{B}} T$ larger than the estimate for shear rate where rotational diffusion becomes important, thermal fluctuations should have little effect on the shear-induced discocyte to prolate transition. The estimate $\dot{\gamma} \tau \simeq 1$ indeed agrees quite well with our simulation results for the shear-induced discocyte to prolate transition, where we find $\dot{\gamma}^{*}=0.1-2$.

The essential factor for the shear-induced shape transformations of Sec. III B is the $\theta$ dependence of the external shear force on the membrane. Shear flow elongates or shrinks a vesicle for $0<\theta<\pi / 2$ or $-\pi / 2<\theta<0$, respectively. These opposite directions of the shear force induce the two types of transformations between discocyte and prolate shapes. When the shear is sufficiently small at $V^{*}=0.59$ and $\eta_{\mathrm{mb}}^{*} \geqslant 0.49$, the shear forces become too small to push the vesicle across the free-energy barrier. Thus, the tumbling prolate should keep its shape and not transform into a disco-

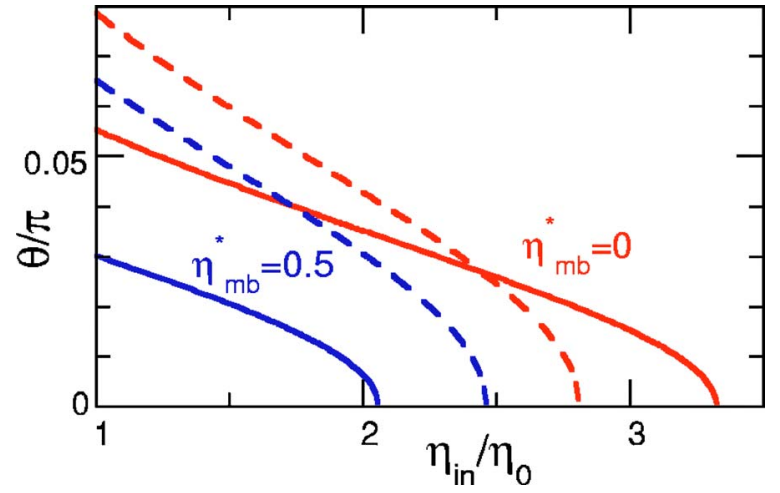

FIG. 14. (Color online) Dependence of the inclination angle $\theta$ on the viscosity $\eta_{\text {in }}$ of an internal fluid at $V^{*}=0.59$ and $\eta_{\mathrm{mb}}^{*}=0$ or 0.5 in KS theory. The solid and dashed lines represent prolate and oblate ellipsoids, respectively.

cyte. We have not been able to investigate this part of the phase diagram by simulations. However, we estimate from Eq. (18) that this behavior occurs for $\dot{\gamma}^{*} \leq 0.1$.

In Eq. (19), only the angular fluctuations in the $x z$ plane are taken into account. This is a reasonable assumption for $\chi \gg 1$. However, at rotational Péclet numbers $\chi \sim 1$, where the transition occurs from random rotational diffusion to tank treading or tumbling, fluctuations in the $y$ direction become important. Thus, our simplified stochastic model is no longer applicable at Péclet numbers $\chi \lesssim 1$.

It is interesting to compare the effects of membrane viscosity $\eta_{\mathrm{mb}}$ and internal viscosity $\eta_{\mathrm{in}}$. It was concluded from the analysis of the behavior of both liquid drops [30] and fluid vesicles of fixed elliptical shape [16] in shear flow that an increase of the surface viscosity has the same qualitative effect as an increase of the internal liquid viscosity $[16,30]$. Indeed, we find for vesicles that an increase of the viscosity induces in both cases a decrease of the inclination angle $\theta$ and a transition from tank treading to tumbling. However, the apparent viscosity obtained from KS theory depends on the vesicle shape, as explained in Appendix B. Therefore, the dependence of the vesicle behavior on $\eta_{\mathrm{mb}}$ and $\eta_{\text {in }}$ can be qualitatively and quantitatively different when shape transformations occur. The effect of the internal viscosity $\eta_{\text {in }}$ is less dependent on the vesicle morphology. KS theory [16] shows that the tank-treading phase of an oblate vesicle is destabilized a little faster than that of a prolate one with increasing $\eta_{\text {in }}$ at $\eta_{\mathrm{mb}}^{*}=0$, but a little slower than that of a prolate at $\eta_{\mathrm{mb}}^{*}=0.5$ (compare Fig. 14). Thus, a sufficiently high membrane viscosity $\eta_{\mathrm{mb}}^{*}$ is necessary to induce the transformation from prolate vesicle to tank-treading discocyte.

When the viscosities $\eta_{\mathrm{mb}}^{*}$ or $\eta_{\mathrm{in}} / \eta_{0}$ are very large, both prolate and discocyte vesicles are in the tumbling phase. On the basis of our simulation results, we expect the following behavior. At large shear rates $\dot{\gamma}^{*}$, tumbling with oscillations between the two shapes should occur. At an intermediate shear rate $\dot{\gamma}^{*}$, there should be a transformation from a tumbling prolate shape to a tumbling discocyte at $V^{*}=0.59$, and from a tumbling discocyte to a tumbling prolate shape at $V^{*}=0.66$. At a small shear rate $\dot{\gamma}^{*}$, no shape transformation should occur, and a tumbling vesicle should keep its initial 
shape. Another factor is that larger viscosities make the shape-transformation rate slower. If the sign of the shear force on the membrane changes during tumbling before the vesicle can overcome the free-energy barrier between discocyte and prolate, the vesicle cannot change its shape. This certainly happens in the asymptotic regime $\eta_{\mathrm{mb}}^{*} \rightarrow \infty$ or $\eta_{\text {in }} / \eta_{0} \rightarrow \infty$, where the vesicle behaves like a solid object.

It would certainly be interesting to extend the present study of vesicles with homogeneous membranes to the case of inhomogeneous membranes, such as phase-separated multicomponent vesicles. If the mechanical properties of the membrane are inhomogeneous in the $x z$ plane, the shape deformation and the inclination angle $\theta$ would oscillate with the tank-treading frequency $\omega / \pi$. Chang and Olbericht [65] report that synthetic capsules exhibit oscillations of the ellipsoidal shape and of the inclination angle $\theta$ in tank-treading motion. These oscillations are likely caused by the inhomogeneity of the membrane.

Since red blood cells have a viscoelastic membrane, their free-energy landscape is different from that of a fluid vesicle (Fig. 2). The shear elasticity suppresses shape changes and prevents the formation of prolate and stomatocyte shapes in the absence of external forces. Pozrikidis $[25,26]$ reported periodic deformation of a tumbling red blood cell in shear flow by the boundary integral method but no shape transitions were obtained.

\section{SUMMARY}

We have applied a mesoscale simulation technique (multiparticle collision dynamics) to the study of the dynamical behavior of vesicles with viscous membranes under shear flow. The membrane viscosity changes the vesicle dynamics from tank treading to tumbling. The shear also induces a discocyte to prolate or prolate to discocyte transformation at low or high membrane viscosity, respectively. Thermal fluctuations modify these transitions. We have explained this effect using a simplified stochastic model based on KellerSkalak theory.

Our model has the advantage that it can easily be adapted to a variety of other problems of vesicle dynamics in flow, like multicomponent vesicles or vesicles with nonzero spontaneous curvature in shear flow, flow of vesicles through capillaries, or vesicles with shear elasticity.

\section{ACKNOWLEDGMENTS}

We would like to thank N. Kikuchi, D. M. Kroll, A. Lamura, K. Mussawisade, M. Ripoll, and R. G. Winkler for helpful discussions. H.N.'s stay at FZJ was supported by the Japan Society for the Promotion of Science (JSPS). G.G. acknowledges partial support of this work by the Deutsche Forschungsgemeinschaft through the priority program "Nano- and Microfluidics."

\section{APPENDIX A: GENERALIZED-ENSEMBLE MONTE CARLO METHOD}

The free energy $F$ of a vesicle is calculated by an orderparameter version of the multicanonical Monte Carlo method [66]. We employ an additional weight potential $U_{\mathrm{w}}$ to obtain

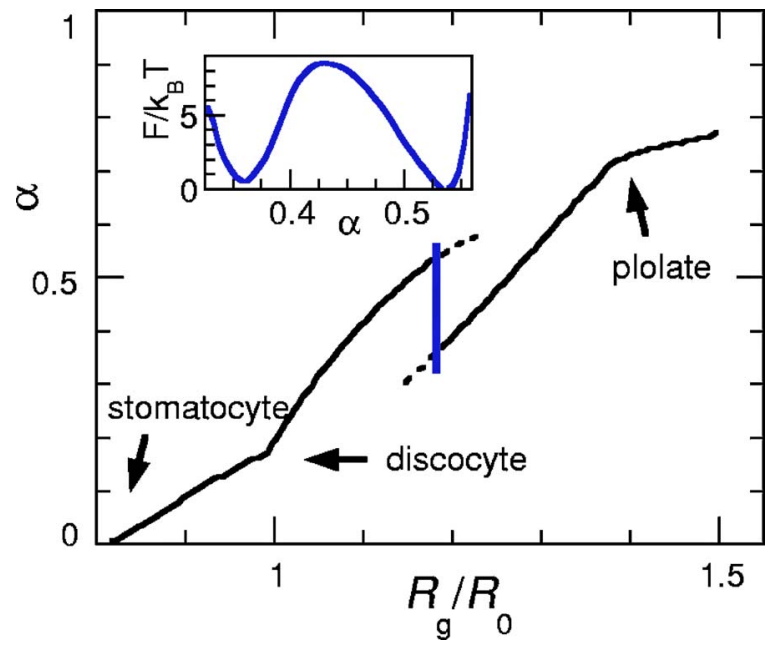

FIG. 15. (Color online) Asphericity $\alpha$ of free-energy minima with each radius of gyration $R_{\mathrm{g}} / R_{0}$ at $V^{*}=0.59$. The solid and dashed lines are the deepest and second minima, respectively. The inset shows the free energy of the asphericity $\alpha$ at $R_{\mathrm{g}} / R_{0}=1.1825$ (along the thick vertical line).

a flat distribution of the order parameters. Here, the reduced radius of gyration $R_{\mathrm{g}}^{*}=R_{\mathrm{g}} / R_{0}$ and the asphericity $\alpha$ are used as order parameters. Since the weight potential $U_{\mathrm{w}}$ is not known a priori, it has to be estimated by an iterative procedure used in the multicanonical method [67-69]. After this simulation, the canonical distribution is obtained by a reweighting procedure [70], $P_{\text {can }} \propto P_{\mathrm{mu}} \exp \left(U_{\mathrm{w}} / k_{\mathrm{B}} T\right)$.

When the vesicle has only one free-energy minimum $\left(V^{*}=0.78\right)$, the free-energy profile $F(\alpha)$ is obtained by a onedimensional weight potential $U_{\mathrm{w}}\left(R_{\mathrm{g}}^{*}\right)$ or $U_{\mathrm{w}}(\alpha)$ (see Fig. 2). At $V^{*}=0.59$, however, the vesicle has a complex free-energy landscape between discocyte and prolate shape, and the onedimensional weight potential does not work. At $V^{*}=0.59$ and 0.66 , we therefore employ a partial two-dimensional weight potential $U_{\mathrm{w}}\left(R_{\mathrm{g}}^{*}, \alpha\right)$. At $V^{*}=0.66$, the two-dimensional weight is more efficient, although the one-dimensional weight also works.

For reduced volume $V^{*}=0.59$, the free energy has three minima, which correspond to stomatocyte, discocyte, and prolate shapes (see Figs. 2 and 15). The vesicle travels between stomatocyte and discocyte shapes with the onedimensional weight, since the free-energy valleys are connected by a single saddle point. On the other hand, the discocyte does not transform into a prolate vesicle and vice versa with the one-dimensional weight. There are freeenergy barriers of about $8 k_{\mathrm{B}} T$ between an elongated discocyte and a banana-shaped prolate vesicle at $R_{\mathrm{g}}^{*} \simeq 1.18$, although they have the same free energy (see inset of Fig. 15). The traffic between these two regions is necessary to estimate the free-energy difference between discocyte and prolate shape. We employ the two-dimensional weight potential $U_{\mathrm{w}}\left(R_{\mathrm{g}}^{*}, \alpha\right)$ in the small region of $1.165 \leqslant R_{\mathrm{g}}^{*}<1.2$ and $\alpha_{\text {prol }}$ $<\alpha<\alpha_{\text {disk }}$, where $\alpha_{\text {prol }}$ and $\alpha_{\text {disk }}$ are the $\alpha$ values of the free-energy minima at each $R_{\mathrm{g}}^{*}$. This two-dimensional weight connects free-energy valleys and enables free-energy estimation. 


\section{APPENDIX B: KELLER-SKALAK THEORY}

In the theory of Keller and Skalak [16], the vesicle is assumed to have a fixed ellipsoidal shape,

$$
\left(x_{1} / a_{1}\right)^{2}+\left(x_{2} / a_{2}\right)^{2}+\left(x_{3} / a_{3}\right)^{2}=1,
$$

where the $a_{i}$ are the semiaxes of the ellipsoid, and the coordinate axes $x_{i}$ point along its principal directions. The $x_{1}$ and $x_{2}$ axes, with $a_{1}>a_{2}$, are chosen to lie in the $x z$ plane. The velocity field on the membrane is assumed to be

$$
\mathbf{v}^{\mathrm{m}}=\omega \mathbf{u}^{\mathrm{m}}=\omega\left(-\left(a_{1} / a_{2}\right) x_{2},\left(a_{2} / a_{1}\right) x_{1}, 0\right) .
$$

Although this velocity field does not satisfy the local area conservation of membrane, it can be analyzed more easily than the area-conserving velocity field of Ref. [17]. The energy $W_{\text {ex }}$ supplied from the external fluid has to be balanced with the energy dissipated in the vesicle, $W_{\mathrm{ex}}=D_{\mathrm{in}}+D_{\mathrm{mb}}$, where $D_{\text {in }}$ and $D_{\mathrm{mb}}$ are the energies dissipated inside the vesicle and on the membrane, respectively. The motion of the vesicle is derived from this energy balance:

$$
\begin{gathered}
\frac{d}{d t} \theta=\frac{1}{2} \dot{\gamma}\{-1+B \cos (2 \theta)\}, \\
B=f_{0}\left\{f_{1}+\frac{f_{1}^{-1}}{1+f_{2}\left(\eta_{\mathrm{in}} / \eta_{0}-1\right)+f_{2} f_{3} \eta_{\mathrm{mb}}^{*}}\right\}, \\
\omega=\frac{0.5 f_{1}^{-1} \dot{\gamma} \cos (2 \theta)}{1+f_{2}\left(\eta_{\mathrm{in}} / \eta_{0}-1\right)+f_{2} f_{3} \eta_{\mathrm{mb}}^{*}} .
\end{gathered}
$$

The membrane-viscosity term has been derived by Tran-SonTay et al. [18]. The factors appearing in Eqs. (B3)-(B5) are given by

$$
\begin{gathered}
f_{0}=2 /\left(a_{1} / a_{2}+a_{2} / a_{1}\right), \\
f_{1}=0.5\left(a_{1} / a_{2}-a_{2} / a_{1}\right), \\
f_{2}=0.5 g\left(\alpha_{1}^{2}+\alpha_{2}^{2}\right), \\
f_{3}=0.5 E_{\mathrm{s}} R_{0} /\left(f_{1}^{2} V\right), \\
g=\int_{0}^{\infty}\left(\alpha_{1}^{2}+s\right)^{-3 / 2}\left(\alpha_{2}^{2}+s\right)^{-3 / 2}\left(\alpha_{3}^{2}+s\right)^{-1 / 2} d s, \\
\alpha_{i}=a_{i} /\left(a_{1} a_{2} a_{3}\right)^{1 / 3}, \\
E_{\mathrm{s}}=\oint \tilde{e}_{i j} \tilde{e}_{i j} d S, \\
\tilde{e}_{i j}=e_{i j}-0.5 \Theta P_{i j}, \\
e_{i j}=0.5 P_{i k} P_{j l}\left(\partial u_{k}^{\mathrm{m}} / \partial x_{l}+\partial u_{l}^{\mathrm{m}} / \partial x_{k}\right), \\
\Theta=P_{i j} \partial u_{i}^{\mathrm{m}} / \partial x_{j}, \\
P_{i j}=\delta_{i j}-n_{i} n_{j},
\end{gathered}
$$

where $E_{\mathrm{s}}$ is an integral over the membrane surface, and $\mathbf{n}$ is the normal vector of the surface.

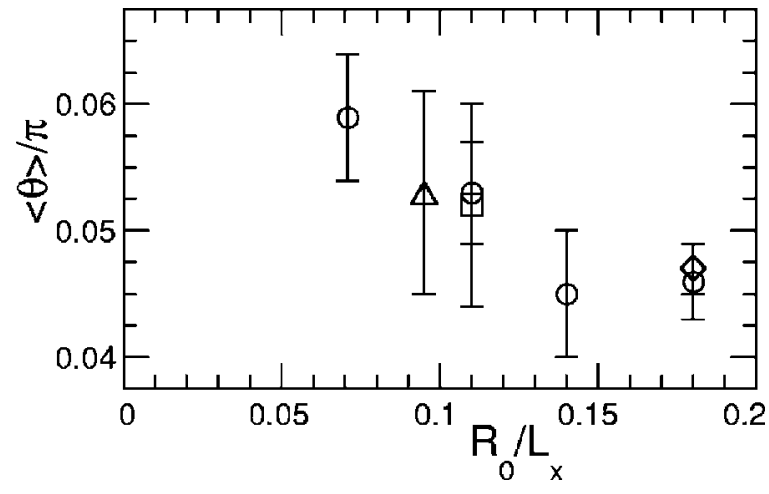

FIG. 16. Finite-size effects of the inclination angle $\langle\theta\rangle$ for $V^{*}$ $=0.59, \eta_{\mathrm{mb}}^{*}=0$, and $\dot{\gamma}=0.92$ in the prolate phase. Circles, diamonds, squares, and triangles represent simulation results for $R_{0} / L_{y}$ $=R_{0} / L_{z}=0.24,0.28,0.19$, and 0.16 , respectively.

For $B>1, \quad$ a steady tank-treading angle $\theta$ $=0.5 \arccos (1 / B)$ is obtained. A simple model, which has been derived by Kraus et al. [20] under the assumption of mirror symmetry of the vesicle shape in the $x z$ plane, gives the same tank-treading angle $\theta=\arctan \left(a_{2} / a_{1}\right)$ of KS theory with $\eta_{\text {in }}=\eta_{0}$ and $\eta_{\mathrm{mb}}^{*}=0$.

We calculate the inclination angle $\theta$ and tank-treading frequency $\omega$ by KS theory for prolate $\left(a_{2}=a_{3}<a_{1}\right)$ and oblate $\left(a_{2}<a_{3}=a_{1}\right)$ ellipsoids. Note that there are no adjustable parameters.

The membrane viscosity $\eta_{\mathrm{mb}}$ and the internal viscosity $\eta_{\text {in }}$ have a similar effect in KS theory. Therefore, an effective internal viscosity can be defined as $\eta_{\text {eff }}^{*}=\eta_{\text {in }} / \eta_{0}+f_{3} \eta_{\text {mb }}^{*}$ from Eqs. (B3)-(B5). Note that the factor $f_{3}$ in $\eta_{\text {eff }}^{*}$ depends on the vesicle shape. This is a significant effect for vesicles of variable shape, in particular for shape transformations between prolate and oblate vesicles (see Fig. 14 and discussion in Sec. V).

\section{APPENDIX C: FINITE-SIZE EFFECTS}

We usually employ a simulation box with $L_{x}=50 a$ and $L_{y}=L_{z}=30 a$ in our simulation. In order to check for finitesize effects, we have also done a few simulations for other box sizes, $32 a \leqslant L_{x} \leqslant 80 a$ and $20 a \leqslant L_{y}=L_{z} \leqslant 36 a$. For a large reduced volume of $V^{*}=0.91$, no significant finite-size effects are observed, since the vesicle is almost spherical and sufficiently far away from its periodic images. On the other hand, for the smallest reduced volume of $V^{*}=0.59$ in our simulations, the long axis of the vesicle reaches about $2 R_{0}$, and the finite-size effect in the flow $(x)$ direction becomes larger. The dependence of the inclination angle $\langle\theta\rangle$ on $R_{0} / L_{x}$ in this case is shown in Fig. 16. The inclination angle is found to increase slightly with increasing $L_{x}$. This size dependence agrees qualitatively with the results for a twodimensional system in Ref. [22]. It implies that we may underestimate the inclination angle by about $10 \%$ for the smallest reduced volume considered. Since this is comparable to our statistical error bars, our box size is sufficiently large for the accuracy of our simulation. 
[1] Structure and Dynamics of Membranes, edited by R. Lipowsky and E. Sackmann (Elsevier Science, Amsterdam, 1995).

[2] U. Seifert, Adv. Phys. 46, 13 (1997).

[3] N. Shahidzadeh, D. Bonn, O. Aguerre-Chariol, and J. Meunier, Phys. Rev. Lett. 81, 4268 (1998).

[4] I. Cantat and C. Misbah, Phys. Rev. Lett. 83, 880 (1999).

[5] S. Sukumaran and U. Seifert, Phys. Rev. E 64, 011916 (2001).

[6] M. Abkarian, C. Lartigue, and A. Viallat, Phys. Rev. Lett. 88, 068103 (2002).

[7] M. M. Guest, T. P. Bond, R. G. Cooper, and J. R. Derrick, Science 142, 1319 (1963).

[8] T. W. Secomb, R. Skalak, N. Özkaya, and J. F. Gross, J. Fluid Mech. 163, 405 (1986).

[9] K. Tsukada, E. Sekizuka, C. Oshio, and H. Minamitani, Microvasc. Res. 61, 231 (2001).

[10] C. Quéguiner and D. Barthès-Biesel, J. Fluid Mech. 348, 349 (1997).

[11] S. Chien, Annu. Rev. Physiol. 49, 177 (1987).

[12] N. Maeda, Jpn. J. Physiol. 46, 1 (1996).

[13] H. L. Goldsmith and J. Marlow, Proc. R. Soc. London, Ser. B 182, 351 (1972).

[14] T. M. Fischer, M. Stöhr-Liesen, and H. Schmid-Schönbein, Science 202, 894 (1978).

[15] K. H. de Haas, C. Blom, D. van den Ende, M. H. G. Duits, and J. Mellema, Phys. Rev. E 56, 7132 (1997).

[16] S. R. Keller and R. Skalak, J. Fluid Mech. 120, 27 (1982).

[17] T. W. Secomb and R. Skalak, Q. J. Mech. Appl. Math. 35(2), 233 (1982).

[18] R. Tran-Son-Tay, S. P. Sutera, and P. R. Rao, Biophys. J. 46, 65 (1984).

[19] T. W. Secomb, T. M. Fischer, and R. Skalak, Biorheology 20, 283 (1983).

[20] M. Kraus, W. Wintz, U. Seifert, and R. Lipowsky, Phys. Rev. Lett. 77, 3685 (1996).

[21] T. Biben and C. Misbah, Phys. Rev. E 67, 031908 (2003).

[22] J. Beaucourt, F. Rioual, T. Séon, T. Biben, and C. Misbah, Phys. Rev. E 69, 011906 (2004).

[23] D. Barthès-Biesel and J. M. Rallison, J. Fluid Mech. 113, 251 (1981).

[24] D. Barthès-Biesel, A. Diaz, and E. Dhenin, J. Fluid Mech. 460, 211 (2002).

[25] C. Pozrikidis, J. Fluid Mech. 440, 269 (2001).

[26] C. Pozrikidis, Ann. Biomed. Eng. 31, 1194 (2003).

[27] G. B. Nash and H. J. Meiselman, Biophys. J. 43, 63 (1983).

[28] B. M. Discher, Y.-Y. Won, D. S. Ege, J. C.-M. Lee, F. S. Bates, D. E. Discher, and D. A. Hammer, Science 284, 1143 (1999).

[29] R. Dimova, U. Seifert, B. Pouligny, S. Förster, and H. G. Döbereiner, Eur. Phys. J. E 7, 241 (2002).

[30] C. Pozrikidis, J. Non-Newtonian Fluid Mech. 51, 161 (1994).

[31] Molecular Gas Dynamics, edited by G. A. Bird (Clarendon, Oxford, 1976).

[32] G. A. Bird, Comput. Math. Appl. 35, 1 (1998).

[33] L. B. Lucy, Astron. J. 82, 1013 (1977).

[34] J. J. Monaghan, Annu. Rev. Astron. Astrophys. 30, 543 (1992).

[35] R. D. Groot and P. B. Warren, J. Chem. Phys. 107, 4423 (1997).

[36] P. Español, Phys. Rev. E 57, 2930 (1998).

[37] H. Noguchi and G. Gompper, Phys. Rev. Lett. 93, 258102 (2004).
[38] A. Malevanets and R. Kapral, J. Chem. Phys. 110, 8605 (1999).

[39] A. Malevanets and R. Kapral, J. Chem. Phys. 112, 7260 (2000).

[40] A. Malevanets and J. M. Yeomans, Europhys. Lett. 52, 231 (2000).

[41] T. Ihle and D. M. Kroll, Phys. Rev. E 63, 020201(R) (2001).

[42] A. Lamura, G. Gompper, T. Ihle, and D. M. Kroll, Europhys. Lett. 56, 319 (2001).

[43] E. Allahyarov and G. Gompper, Phys. Rev. E 66, 036702 (2002).

[44] T. Sakai, Y. Chen, and H. Ohashi, Phys. Rev. E 65, 031503 (2002).

[45] T. Ihle and D. M. Kroll, Phys. Rev. E 67, 066705 (2003).

[46] T. Ihle and D. M. Kroll, Phys. Rev. E 67, 066706 (2003).

[47] E. Tüzel, M. Strauss, T. Ihle, and D. M. Kroll, Phys. Rev. E 68, 036701 (2003).

[48] N. Kikuchi, C. M. Pooley, J. F. Ryder, and J. M. Yeomans, J. Chem. Phys. 119, 6388 (2003).

[49] N. Kikuchi, Ph.D. thesis, University of Oxford, 2003 (unpublished).

[50] M. Ripoll, K. Mussawisade, R. G. Winkler, and G. Gompper, Europhys. Lett. 68, 106 (2004).

[51] E. Falck, J. M. Lahtinen, I. Vattulainen, and T. Ala-Nissila, Eur. Phys. J. E 13, 267 (2004).

[52] K. Tucci and R. Kapral, J. Chem. Phys. 120, 8262 (2004).

[53] A. Lamura and G. Gompper, Eur. Phys. J. E 9, 477 (2002).

[54] M. P. Allen and D. J. Tildesley, Computer Simulation of Liquids (Clarendon Press, Oxford, 1987).

[55] G. Gompper and D. M. Kroll, J. Phys.: Condens. Matter 9, 8795 (1997).

[56] G. Gompper and D. M. Kroll, in Statistical Mechanics of Membranes and Surfaces, edited by D. R. Nelson, T. Piran, and S. Weinberg, 2nd ed. (World Scientific, Singapore, 2004).

[57] W. Helfrich, Z. Naturforsch [C] 28C, 693 (1973).

[58] C. Itzykson, in Proceedings of the GIFT seminar, Jaca 85, edited by J. Abad, M. Asorey, and A. Cruz (World Scientific, Singapore, 1986).

[59] G. Gompper and D. M. Kroll, J. Phys. I 6, 1305 (1996).

[60] F. H. Stillinger and T. A. Weber, Phys. Rev. B 31, 5262 (1985).

[61] J. Rudnick and G. Gaspari, J. Phys. A 19, L191 (1986).

[62] H. Noguchi and K. Yoshikawa, J. Chem. Phys. 109, 5070 (1998).

[63] U. Seifert, K. Berndl, and R. Lipowsky, Phys. Rev. A 44, 1182 (1991).

[64] P. Bergé, Y. Pomeau, and C. Vidal, Order within Chaos: Towards a Deterministic Approach to Turbulence (Wiley, New York, 1984).

[65] K. S. Chang and W. L. Olbricht, J. Fluid Mech. 250, 609 (1993).

[66] B. A. Berg, H. Noguchi, and Y. Okamoto, Phys. Rev. E 68, 036126 (2003).

[67] B. A. Berg and T. Neuhaus, Phys. Lett. B 267, 249 (1991).

[68] B. A. Berg, Comput. Phys. Commun. 153, 397 (2003).

[69] U. H. E. Hansmann and Y. Okamoto, Phys. Rev. E 56, 2228 (1997).

[70] A. M. Ferrenberg and R. H. Swendsen, Phys. Rev. Lett. 61, 2635 (1988). 